\title{
Endometrial Cancer
}

\author{
Mariana Horta and Teresa Margarida Cunha
}

\section{Contents}

1 Endometrial Cancer: Background ............. 000

1.1 Epidemiology ............................................... 000

1.2 Pathology and Risk Factors........................... 000

1.3 Symptoms and Diagnosis.............................. 000

2 Endometrial Cancer Staging ....................... 000

2.1 MR Protocol for Staging Endometrial

Carcinoma

2.2 MR Findings According to the Stage of Endometrial Carcinoma

3 Recent Advances in Functional

MR Imaging in Assessing

Endometrial Carcinoma

4 Therapeutic Approaches.

000

4.1 Surgery

4.2 Adjuvant Treatment

4.3 Fertility-Sparing Treatment

000

000

5 Follow-Up and Recurrent Endometrial Carcinoma

5.1 Treatment of Recurrence. 000

6 Prognosis 000

Conclusion 000

References 000

\footnotetext{
M. Horta ( $\square)$

Centro Hospitalar Lisboa Ocidental,

Estrada do Forte do Alto do Duque,

1495-005 Lisbon, Portugal

e-mail: mariana.sf.horta@gmail.com

T.M. Cunha

Rua Professor Lima Basto, 1099-023 Lisbon, Portugal e-mail: tmargarida@gmail.com
}

\section{Abstract}

Endometrial cancer is the most common gynecological malignancy in well-developed countries. Biologically and clinicopathologically, endometrial carcinomas are divided into two types: type 1 or estrogen-dependent carcinomas and type 2 or estrogen-independent carcinomas. Type 1 cancers correspond mainly to endometrioid carcinomas and account for approximately $90 \%$ of endometrial cancers, whereas type 2 cancers correspond to the majority of the other histopathological subtypes.

The vast majority of endometrial cancers present as abnormal vaginal bleedings in postmenopausal women. Therefore, $75 \%$ of cancers are diagnosed at an early stage, which makes the overall prognosis favorable.

The first diagnostic step to evaluate women with an abnormal vaginal bleeding is the measurement of the endometrial thickness with transvaginal ultrasound. If endometrial thickening or heterogeneity is confirmed, a biopsy should be performed to establish a definite histopathological diagnosis.

Magnetic resonance imaging is not considered in the International Federation of Gynaecology and Obstetrics staging system. Nonetheless it plays a relevant role in the preoperative staging of endometrial carcinoma, helping to define the best therapeutic management. Moreover, it is important in the diagnosis of treatment complications, in the surveillance of therapy response, and in the assessment of recurrent disease. 


\section{Endometrial Cancer: Background}

\section{$1.1 \quad$ Epidemiology}

Endometrial cancer is the fifth most common malignancy in females worldwide, after breast, colorectal, cervical, and lung cancers, accounting for $4.8 \%$ of all cancers in women (Ferlay et al. 2013).

In contrast to cervical cancer, endometrioid cancer peak incidence rates occur in well-developed countries (Europe and North America), where it is the most common gynecological malignancy (Ferlay et al. 2013). This is probably due to lifestyle factors, in particular obesity, which has been linked to about $50 \%$ endometrial cancers in these countries (Epstein and Blomqvist 2014; Calle and Kaaks 2004). The increase in life expectancy is also responsible for its rising (Koh et al. 2014).

Endometrial cancer is more likely to occur in postmenopausal women in their sixth and seventh decades, with cases only rarely reported under the age of 35 in the United Kingdom (Patel et al. 2010; Cancer Incidence Statistics 2015).

Despite its relatively high prevalence, approximately $82 \%$ of endometrial cancers are diagnosed at an early stage, which makes its overall prognosis favorable (Cancer Incidence Statistics 2015). Its worldwide mortality rate is low and it has been estimated at $2.2 \%$ (Ferlay et al. 2013).

\subsection{Pathology and Risk Factors}

The World Health Organization (WHO) classification of uterine corpus tumors was revised in 2014 (Kurman et al. 2014). Endometrial carcinomas (also known as adenocarcinoma of the endometrium) are classified under the "Epithelial Tumours and Precursors" group and clinicopathologically divided into the following types: endometrioid carcinoma; mucinous carcinoma; serous endometrial intraepithelial carcinoma; serous carcinoma; clear-cell carcinoma; neuroendocrine tumors; mixed cell adenocarcinoma; undifferentiated carcinoma; and dedifferentiated carcinoma (Table 1) (Kurman et al. 2014).

Although carcinosarcoma belongs to the "Mixed Epithelial and Mesenchymal Tumours"
Table 1 World Health Organization classification of epithelial tumors and precursors of the uterine corpus

\begin{tabular}{l}
\hline Precursors \\
\hline Hyperplasia without atypia \\
\hline $\begin{array}{l}\text { Atypical hyperplasia/endometrioid intraepithelial } \\
\text { neoplasia }\end{array}$ \\
\hline Endometrial cancer \\
\hline Endometrioid carcinoma \\
\hline Squamous differentiation \\
\hline Villoglandular \\
\hline Secretory \\
\hline Mucinous carcinoma \\
\hline Serous endometrial intraepithelial carcinoma \\
\hline Serous carcinoma \\
\hline Clear-cell carcinoma \\
\hline Neuroendocrine tumors \\
\hline Low-grade neuroendocrine tumor (carcinoid \\
tumor) \\
\hline $\begin{array}{l}\text { High-grade neuroendocrine carcinoma (small cell } \\
\text { neuroendocrine carcinoma; large cell } \\
\text { neuroendocrine carcinoma) }\end{array}$ \\
\hline Mixed cell adenocarcinoma \\
\hline Undifferentiated carcinoma \\
\hline Dedifferentiated carcinoma \\
\hline
\end{tabular}

Adapted from Kurman et al. (2014)

group of the uterine corpus, it should be staged as an endometrial carcinoma (Kurman et al. 2014).

Biologically and clinicopathologically, endometrial carcinomas are divided into two types: type 1 or estrogen-dependent carcinomas and type 2 or estrogen-independent carcinomas (Table 2).

Type 1 correspond mainly to endometrioid carcinomas and accounts for approximately $90 \%$ of endometrial cancers, whereas type 2 cancers correspond to the majority of the other histopathological subtypes (Epstein and Blomqvist 2014).

Type 1 endometrial cancers are associated with prolonged unopposed estrogen exposure, therefore with the following risk factors: estrogen replacement therapy; tamoxifen therapy for breast cancer; polycystic ovarian syndrome; estrogen producing ovarian tumors; obesity (conversion of androgen to estrone in adipose tissue); diabetes; early menarche and late menopause and nulliparity (Shapiro et al. 1985; Fisher et al. 1994; Renehan et al. 2008; Soliman et al. 2006; McPherson et al. 1996).

Endometrioid cancers are endometrial glandular neoplasms that can be preceded or associated 
Table 2 Types of endometrial cancer

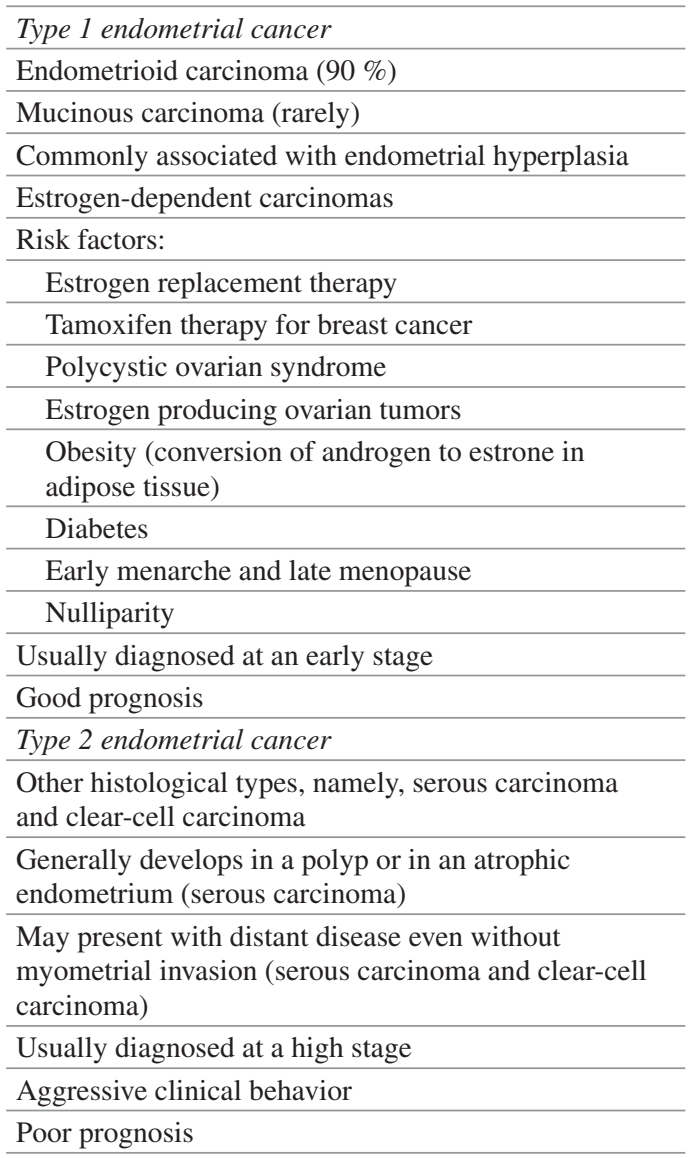

with endometrial hyperplasia (Kurman et al. 2014; Colombo et al. 2013). They are subdivided into three grades of differentiation (well to poorly differentiated) according to the amount of solid components (Colombo et al. 2013; Tirumani et al. 2013). Low-grade tumors are usually diagnosed at an early stage and generally have a good outcome, whereas high-grade endometrioid cancers are associated with a poor prognosis (Tirumani et al. 2013).

Endometrioid cancers are characteristically associated with PTEN, K-RAS, and CTNNB gene mutations (Tirumani et al. 2013).

Mucinous carcinomas are a rare form of type 1 endometrial cancer, where more than $50 \%$ of glandular cells mucin can be found. They are in the majority of times well differentiated and associated with good prognosis (Kurman et al. 2014; Jalloul et al. 2012).

Type 2 tumors are constituted by the other histological types, namely, serous carcinoma and clear-cell carcinoma (Tirumani et al. 2013; Jalloul et al. 2012). They have a more aggressive clinical behavior, a poorer prognosis, and are usually diagnosed at higher stage (Tirumani et al. 2013; Jalloul et al. 2012).

Genetics changes such as p53 and p16 inactivation genes and gene overexpression of HER-2/neu are frequently present in these types of carcinomas (Tirumani et al. 2013; Hecht and Mutter 2006).

Although classically considered estrogenindependent cancers, Setiawan and her colleagues found an overlap between risk factors of type 1 and type 2 endometrial cancers (Setiawan et al. 2013). Parity, oral contraceptives use, age at menarche, diabetes and smoking were associated with both type 2 (mainly serosal and mixed carcinomas) and type 1 cancers (Setiawan et al. 2013). The risk factors of type 2 endometrial cancer and high-grade endometrioid tumors were similar, whereas body mass index was more associated with type 1 cancer (Setiawan et al. 2013).

Serous carcinomas make up the majority of type 2 endometrial cancers. They are characterized by the presence of complex papillary architecture associated with remarkable nuclear pleomorphism (Kurman et al. 2014). They are not associated with endometrial hyperplasia, but may be preceded by serous endometrial intraepithelial carcinoma, a noninvasive form of carcinoma confined to the epithelium that generally develops in a polyp or in an atrophic endometrium (Kurman et al. 2014).

Clear-cell carcinoma is a rare form of type 2 cancer that resembles its ovarian counterpart (Kurman et al. 2014).

Undifferentiated and dedifferentiated carcinomas of the endometrium are also rare types. The former is characterized by the presence of cells with no differentiation, whereas the latter is composed partially by undifferentiated type carcinoma and by International Federation of Gynaecology Obstetrics (FIGO) grade 1 or 2 endometrioid carcinoma (Kurman et al. 2014).

Mixed carcinomas are made of two or more types of endometrial carcinomas, with at least one being a type 2 carcinoma (Kurman et al. 2014).

Neuroendocrine tumors of the endometrium are extremely rare tumors. 
Although they present different pathological and epidemiological features, in the latest WHO classification they have been grouped under endometrial carcinomas (Kurman et al. 2014).

Endometrial neuroendocrine tumors may be of low grade (carcinoid tumors) or of high grade (small cell neuroendocrine carcinomas and large cell neuroendocrine tumors).

The majority of cases reported in the literature are of small cell neuroendocrine carcinomas, which present as their lung counterpart (Lopes Dias et al. 2015).

They tend to be aggressive tumors with a poor prognosis. Women are generally in the postmenopausal age and tend to present with vaginal bleeding, but sometimes due to their aggressiveness, metastatic pain can be present (Lopes Dias et al. 2015; Eichhorn and Young 2001).

Carcinosarcomas are an admixture of carcinomatous and mesenchymal sarcomatous components (Kurman et al. 2014). Carcinomatous elements are generally high-grade endometrioid, serous, clear-cell, or undifferentiated carcinomas. Carcinosarcomas are classified under the "Mixed epithelial and mesenchymal" group (Kurman et al. 2014). However, due to their carcinomatous component and to the fact that their risk factors present symptoms and behavior similar to those of high-grade endometrial carcinomas, they are staged as such.

The radiological and pathological appearances of these tumors tend to differ from the other histopathological endometrial cancer subtypes, as they tend to present as large hemorrhagic and necrotic masses that distend the endometrial cavity, compressing and frequently invading the myometrium and the cervical stroma. Their prognosis is poor since they tend to present lymphatic and sometimes hematogenous spread at the time of diagnosis. Recurrence is also frequent.

The majority of endometrial cancers are sporadic. Nonetheless, about $5 \%$ of endometrial cancers are caused by genetic mutations (e.g., Lynch syndrome, Cowden syndrome). These cancers tend to occur 10-20 years before the sporadic type (Koh et al. 2014; Resnick et al. 2009).

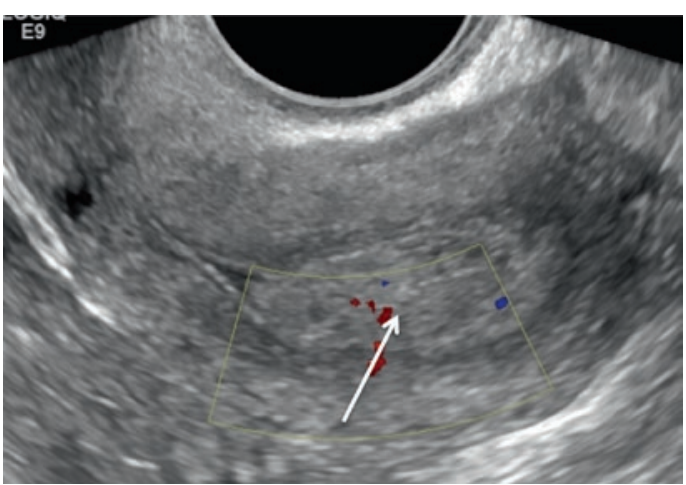

Fig. 1 Serous endometrial carcinoma in a 59-year-old woman presenting with vaginal bleeding. Transvaginal ultrasonography detected thickening of the endometrium (arrow), which was posteriorly submitted to biopsy that diagnosed a serous endometrial carcinoma

\subsection{Symptoms and Diagnosis}

About $90 \%$ of patients with endometrial carcinoma present with abnormal vaginal bleeding (Koh et al. 2014; Colombo et al. 2013). Therefore, endometrial carcinoma is often diagnosed at an early and treatable stage, at which the prognosis is good.

Some women also seek care owing to pelvic pain or pressure (usually patients with advanced stage disease). Moreover, patients may present with atypical glandular cells on cervical cytology, which requires further evaluation for premalignant or malignant diseases of the endocervix and of the endometrium.

The first diagnostic step to evaluate women with pre- and postmenopausal abnormal vaginal bleeding should be the measurement of endometrial thickness with transvaginal ultrasound (TVUS) (Fig. 1) (Bennet et al. 2011). Transabdominal ultrasound may also be used as an adjunct modality, particularly in large leiomyomatous uterus or when women cannot tolerate TVUS (Bennet et al. 2011).

In postmenopausal women with vaginal bleeding an upper threshold of $5 \mathrm{~mm}$ or of $4 \mathrm{~mm}$ for normal endometrial thickness should be considered (Bennet et al. 2011; Gull et al. 2003; Karlsson et al. 1995; Smith-Bindman et al. 1998). In this age group, an endometrial thickness measurement 
$\leq 5 \mathrm{~mm}$ is associated with the absence of endometrial cancer (Gupta et al. 2002). This cut-off value should also be used in women under hormone replacement therapy and under tamoxifen (Bennet et al. 2011).

In symptomatic patients in premenopausal age, an upper threshold of normal endometrial thickness is more difficult to establish due to its variations during the menstrual cycle. In this group of women, the assessment of the endometrium should be performed during the early first half of the menstrual cycle (Bennet et al. 2011). In these women, an endometrial thickness $>16 \mathrm{~mm}$ has shown to have a sensitivity of $67 \%$ and a specificity of $75 \%$ for detecting endometrial abnormalities (Bennet et al. 2011; Hulka et al. 1994). A heterogeneous endometrium or an area of focal thickness should be also further investigated (Bennet et al. 2011; Goldstein et al. 2001).

If the endometrium is thickened or heterogeneous, a sample of the endometrium must be undertaken to establish a definite histopathological diagnosis. In these cases a hysteroscopy with biopsy or resection should be performed.

Before treatment, the initial evaluation should include a medical history, a physical and gynecological examination, a complete blood count, and a chest radiograph (Koh et al. 2014).

If the age of the onset of the disease is $<50$ years and if there is history of familiar colorectal or endometrial cancer, screening for genetic mutations should be performed (Koh et al. 2014).

Women with Lynch syndrome, without endometrial cancer, should be screened annually with an endometrial biopsy (Koh et al. 2014; Järvinen et al. 2009; Meyer et al. 2009). Prophylactic hysterectomy with bilateral salpingoophorectomy may be considered in these patients (Koh et al. 2014; Schmeler et al. 2006).

\section{Endometrial Cancer Staging}

Endometrial carcinoma is staged with the International Federation of Gynaecology Obstetrics (FIGO) system, which was last revised
Table 3 Endometrial Cancer Staging: FIGO 2009

Stage I-The tumor is confined to the uterine corpus

IA-Absence or invasion of $<50 \%$ of the

myometrium

IB-Invasion of $\geq 50 \%$ of the myometrium

Stage II-The tumor invades the cervical stroma, but not beyond the uterus

Stage III-There is local or regional involvement

IIIA - The tumor invades the serosa and/or the adnexa

IIIB-Presence of vaginal and/or parametrial

involvement

IIIC_-Presence of pelvic or para-aortic

lymphadenopathies

$\mathrm{III}_{\mathrm{C} 1}$-Presence of pelvic lymphadenopathies

$\mathrm{III}_{\mathrm{C} 2}$-Presence of para-aortic lymphadenopathies,

with or without pelvic lymphadenopathies

Stage IV-The tumor invades the bladder mucosa and/ or the intestinal mucosa, and/or there are distant metastases

IVA-The tumor invades the bladder mucosa and/or the intestinal mucosa

IVB-Presence of distant metastases, including abdominal metastases and/or inguinal lymphadenopathies.

Adapted from Pecorelli (2009)

${ }^{\mathrm{a}} \mathrm{FIGO}$ International Federation of Gynaecology and Obstetrics

in 2009 (Table 3) (Creasman 2009; Pecorelli 2009). This classification defines that endometrial carcinoma is staged on the basis of surgicopathological findings.

The complete surgical staging procedure implies hysterectomy with bilateral salpingooophorectomy, assessment of the abdominal cavity with biopsies of suspicious peritoneal lesions, cytology of peritoneal washings, and pelvic and retroperitoneal lymphadenectomy (Koh et al. 2014). In patients with serous carcinoma, clearcell carcinoma, and carcinosarcoma, the surgical staging procedure should be the same as for ovarian cancer (Koh et al. 2014).

The FIGO 2009 staging classification is as follows (Table 3) (Pecorelli 2009):

In stage I, the tumor is confined to the uterus. This stage is subdivided in two substages: stage IA (the tumor invades $<50 \%$ of the myometrium) and stage IB (the tumor invades $\geq 50 \%$ of the myometrium). 
In stage II, the tumor invades the cervical stroma, but not beyond the uterus.

Stage III is subdivided into three substages. In stage IIIA, the tumor invades the serosa or/and the adnexa (direct extension or metastasis). In stage IIIB, there is involvement of the parametria and/or the vagina (direct extension or metastasis). In stage IIIC, there is lymph node involvement $\left(\mathrm{III}_{\mathrm{Cl}}\right.$ if there are positive pelvic lymph nodes and $\mathrm{III}_{\mathrm{C} 2}$ if there are positive para-aortic lymph nodes).

Stage IV is also subdivided into two substages. The tumor is in stage IVA if there is mucosal invasion of the bladder or/and the bowel and it is in stage IVB if there are distant metastasis including abdominal metastasis and/or the presence of positive inguinal lymph nodes.

MR imaging is not considered in the FIGO staging of endometrial carcinoma, but due to its high contrast resolution and reproducibility, it has an important role in the preoperative staging of the disease. Thus, it is crucial for tailoring the surgical approach to these patients.

There is still no consensus on whether complete surgical staging with primary pelvic and para-aortic lymphadenopathy should be performed at stage I, namely, in patients with low recurrence risk (Bonatti et al. 2015; Todo et al. 2010; May et al. 2010). However, it is known that the tumor histological type and grade, the presence of myometrial invasion $\geq 50 \%$, and the presence of lymphovascular space invasion correlate with the presence of lymph node metastasis and with overall survival (Rechichi et al. 2010; Sala et al. 2013; Boronow 1990; Larson et al. 1996). From these features, only the histological type and grade can be assessed preoperatively without imaging. Nonetheless, discrepancies of up to $15 \%$ between the pre- and postoperative tumoral histopathological results have been described (Sala et al. 2013; Frei et al. 2000).

MR can accurately determine the depth of myometrial invasion. Therefore, in conjunction with the histopathological grade it may be used to select the patients that might be candidates for pelvic and para-aortic lymphadenectomy, precluding surgery in low-risk patients, thus avoiding the morbidities associated with this procedure.
Presence of lymph node metastases has been reported in more than $30 \%$ of the cases of endometrial cancer with $\geq 50 \%$ of myometrial invasion and in only $5 \%$ of cases when the tumor invades $<50 \%$ of the myometrium (Larson et al. 1996; Gallego et al. 2014).

Lymphovascular invasion is generally assessed postoperatively. It is related not only to the likelihood of lymph node involvement, but with tumor relapse and poor survival (Sala et al. 2013; Fujii et al. 2015; Briët et al. 2005). Not many studies have addressed the role of MR in diagnosing lymphovascular space involvement. However, a study by Nougaret et al. showed that whole tumor volume and ADC could be useful in its prediction (Nougaret et al. 2015).

Cervical stromal invasion is also associated with lymph node metastasis and poor survival. MR can accurately diagnose cervical stromal invasion and parametrial invasion. This is particularly important so surgeons can avoid cutting through the tumor and thus perform an extensive resection (Sala et al. 2013).

Moreover, MR is helpful is diagnosing advanced disease involving the adnexa and the peritoneum, which generally are contraindications to laparoscopic and robotic surgery (Sala et al. 2013; Venkat et al. 2012; Amant et al. 2007). Other extra-uterine coexistent pathologies can also be diagnosed that may help determining the surgical approach.

Therefore, MR is useful not only in planning surgical treatment but also in selecting more advanced and difficult surgical cases that should be guided to specialized oncologic centers.

MR is also useful in determining the origin (cervical or endometrial) of a biopsy proven adenocarcinoma (Fig. 2). Vargas and colleagues showed that by assessing the epicenter of the tumor, the endometrial versus the cervical origin of an adenocarcinoma could be determined with an accuracy of 85-88 \% (Vargas et al. 2011). Furthermore, endometrial thickening, expansion of the endometrial cavity by a mass, and the presence of a tumor invading the myometrium may aid the discrimination between these two types of tumors (Haider et al. 2006). This distinction is particularly important, because early stage cervical adenocarcinomas are 


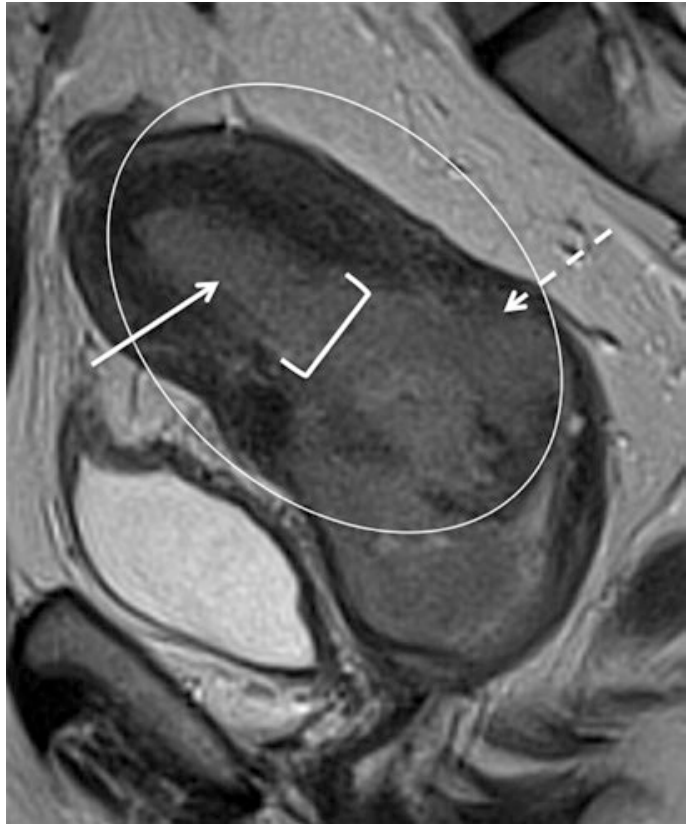

Fig. 2 Endometrial carcinoma that invades the cervical stroma in a 53-year-old woman. Sagittal T2-weighted image. Distinction between endometrial and cervical origin of an adenocarcinoma may be challenging. Radiologists should look for the epicenter of the tumor $(>50 \%)$ (circle), and other signs that may help defining endometrial origin, such as endometrial thickness (arrow), expansion of the uterine cavity by a mass (squared brackets), and invasion of the myometrium (dashed arrow)

treated with surgery, advanced stage cervical adenocarcinomas are treated with chemotherapy and radiotherapy and endometrial carcinomas are treated primarily with surgery.

Although fertility-sparing progesterone therapy is not the standard of care for endometrial cancers, it might be considered according to patients request in treatment of low-grade endometrial cancers that do not invade the myometrium. In these cases, MR is essential in defining which patients are candidates to this approach.

\subsection{MR Protocol for Staging Endometrial Carcinoma}

In our institution the MR protocol for staging endometrial carcinoma is as follows (Table 4):

Patients are advised to fast $4-6 \mathrm{~h}$ before the procedure. A fast acting laxative enema to clean
Table 4 MR protocol scheme for endometrial cancer staging

\begin{tabular}{|c|}
\hline \multirow{2}{*}{$\begin{array}{l}\text { MRI protocol for endometrial cancer staging } \\
\text { Abdomen }\end{array}$} \\
\hline \\
\hline $\begin{array}{l}\text { T2 FSE axial }(6 \mathrm{~mm} / 1 \mathrm{~mm}) \text { - from the diaphragm } \\
\text { to the iliac crests }\end{array}$ \\
\hline Axial DWI and the respective ADC maps (6 mm) \\
\hline Pelvis \\
\hline T1 FSE axial $(5 \mathrm{~mm} / 0.5 \mathrm{~mm})$ \\
\hline $\begin{array}{l}\text { T2 FSE axial }(5 \mathrm{~mm} / 0.5 \mathrm{~mm}) \text {; T2 FSE sagittal } \\
(4 \mathrm{~mm} / 0.4 \mathrm{~mm}) \text {; T2 FSE axial oblique of the uterine } \\
\text { corpus (perpendicular to the uterine cavity; } \\
4 \mathrm{~mm} / 0.4 \mathrm{~mm})\end{array}$ \\
\hline $\begin{array}{l}\text { Dynamic contrast-enhanced study in the axial } \\
\text { oblique plane of the uterine corpus: 3D T1, FS } \\
\text { pre- and postcontrast administration, five } \\
\text { acquisition phases (until } 150 \mathrm{~s} ; 2 \mathrm{~mm} \text { ) }\end{array}$ \\
\hline $\begin{array}{l}\text { Axial DWI and the respective ADC maps } \\
(5 \mathrm{~mm} / 0.5 \mathrm{~mm})\end{array}$ \\
\hline Protocol variants \\
\hline If there is suspicion of cervix invasion: \\
\hline $\begin{array}{l}\text { T2 FSE axial oblique of the uterine cervix } \\
\text { (perpendicular to the cervical canal; } 4 \mathrm{~mm} / 0.4 \mathrm{~mm} \text { ) }\end{array}$ \\
\hline $\begin{array}{l}\text { Dynamic contrast-enhanced study in the sagittal } \\
\text { plane: 3D T1, FS pre- and postcontrast } \\
\text { administration, five acquisition phases (until } 150 \mathrm{~s} \text {; } \\
2 \mathrm{~mm} \text { ) }\end{array}$ \\
\hline $\begin{array}{l}\text { Axial oblique plane of the uterine cervix } \\
\text { postcontrast injection } 3 \mathrm{D} \text { T1, FS in late phase } \\
(4 \mathrm{~min} ; 2 \mathrm{~mm})\end{array}$ \\
\hline
\end{tabular}

the bowel is administered the day before the exam and during the morning of the exam. Patients are encouraged to void before the examination since a full bladder degrades the quality of T2-weighted images.

In order to reduce bowel peristalsis and hence improve image quality, $1 \mathrm{mg}$ of glucagon intravenous is administered before the exam. Alternatively, $20 \mathrm{mg}$ or $40 \mathrm{mg}$ of hyoscine butylbromide intramuscular/intravenous can be administered.

Patients are advised to avoid the use of vaginal tampon.

MR imaging can either be performed in a $1.5 \mathrm{~T}$ or a $3 \mathrm{~T}$ MR machine. $3 \mathrm{~T}$ MR has been not shown to offer significant benefit over 1.5 T MR in the evaluation of endometrial cancer, since $3 \mathrm{~T}$ MR is more prone to susceptibility and chemicalshift artifacts that impair the image quality (Wakefield et al. 2013; Haldorsen and Salvesen 2012; Hori et al. 2013). 

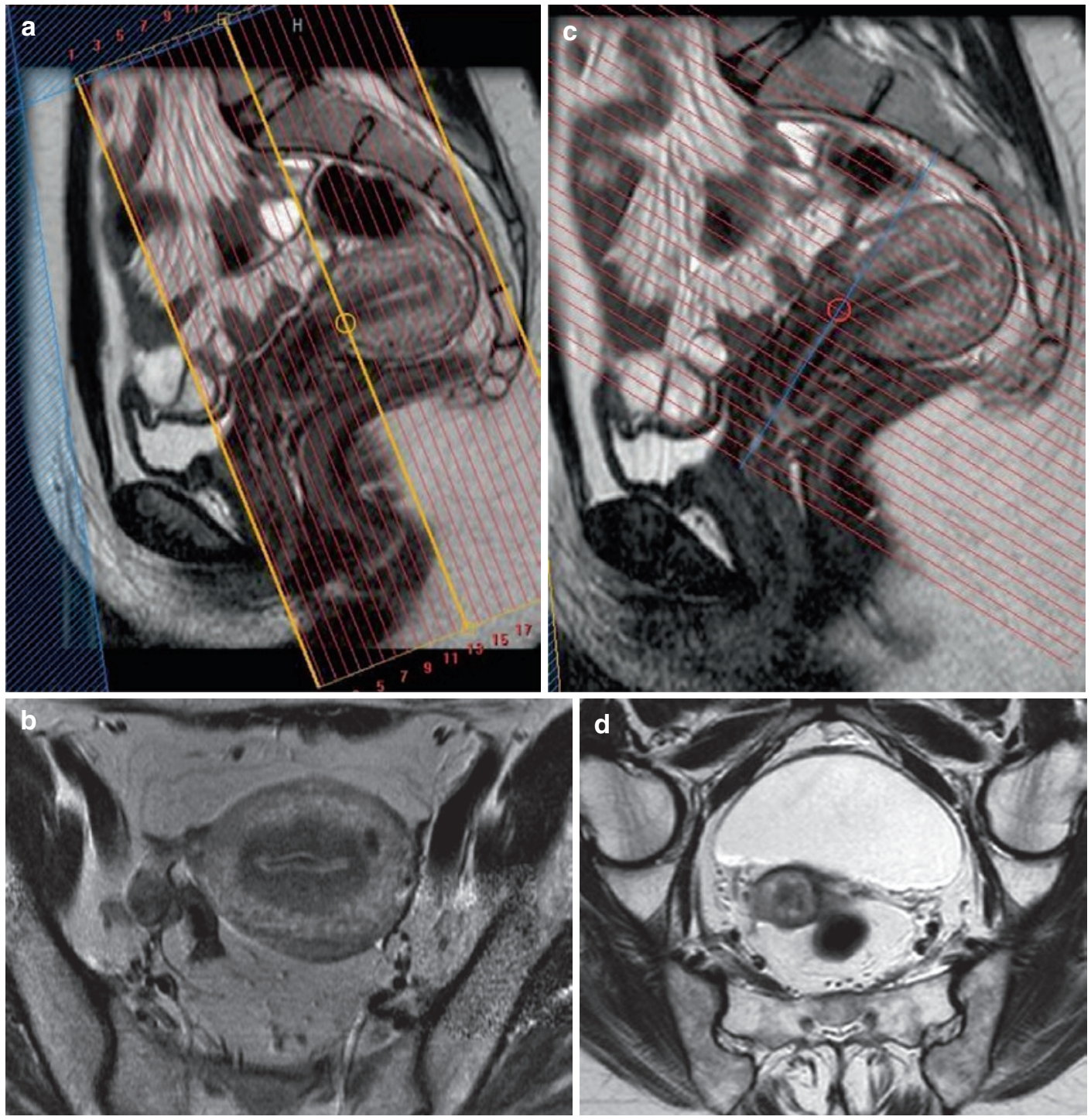

Fig. 3 (a, b) Axial oblique plane of the uterine corpus (perpendicular to the uterine cavity); (c, d) axial oblique plane of the uterine cervix (perpendicular to the cervical canal)

A pelvic phase array is used along with anterior and superior saturation bands.

The patient should be placed in a supine position for the whole exam. However, prone position may be used in uncooperative patients.

Axial imaging of the abdomen (from diaphragm to iliac crests) should be performed for the evaluation of advanced disease, with fast recovery, fast spin-echo T2weighted images $(6 \mathrm{~mm} / 1 \mathrm{~mm}$, breath-hold). Abdominal axial diffusion-weighted images (DWI)
(6 mm, b-values- $\left.0,500,1000 \mathrm{~s} / \mathrm{mm}^{2}\right)$ and the respective ADC maps should also be obtained.

Evaluation of the pelvis should be performed with axial fast spin-echo T1-weighted images ( $5 \mathrm{~mm} / 0.5 \mathrm{~mm})$, axial fast spin-echo T2-weighted images $(5 \mathrm{~mm} / 0.5 \mathrm{~mm})$, and sagittal fast spinecho T2-weighted images ( $4 \mathrm{~mm} / 0.4 \mathrm{~mm})$.

Fast spin-echo T2-weighted axial oblique images (perpendicular to the uterine cavity, $4 \mathrm{~mm} / 0.4 \mathrm{~mm}$ ) are helpful in assessing myometrial invasion (Fig. 3). 
The dynamic contrast-enhanced MR images are obtained after the injection of $0.1 \mathrm{mmol} / \mathrm{kg}$ of gadolinium at a rate of $2 \mathrm{~mL} / \mathrm{s}$. In our institution, images are acquired using a 3D-recalled echo fatsuppressed T1-weighted sequence, pre- and postcontrast injection during five acquisition phases in the axial-oblique plane (perpendicular to the uterine cavity), until $150 \mathrm{~s}$ ( $2 \mathrm{~mm}$ ). The dynamic study tends to be avoided in patients with renal impairment.

DWI study (b-values-0, 600, $1000 \mathrm{~s} / \mathrm{mm}^{2}$, $5 \mathrm{~mm} / 0.5 \mathrm{~mm}$ ) and the respective ADC maps are performed in the axial plane.

If there is suspicion of cervix invasion, axial oblique of the cervix fast spin-echo T2-weighted images (perpendicular to the cervical canal, $4 \mathrm{~mm} / 0.4 \mathrm{~mm}$ ) are obtained to evaluate parametrial invasion (Fig. 3). In these cases, the dynamic study should be performed in the sagittal plane using a 3D-reccaled echo fat-suppressed T1-weighted sequence, pre- and postcontrast injection during five acquisition phases until $150 \mathrm{~s}(2 \mathrm{~mm})$. Furthermore, axial oblique of the cervix (perpendicular to cervical canal) postcontrast injection 3D-reccaled echo fat-suppressed T1-weighted images are obtained in a late phase (4 min, $2 \mathrm{~mm}$ ), to better assess cervical stromal invasion (Fig. 3).

\subsection{MR Findings According to the Stage of Endometrial Carcinoma}

On conventional imaging, the normal uterus anatomy is better depicted on T2-weighted images. The endometrium tends to be hyperintense, the junctional zone is hypointense and the outer myometrium shows intermediate intensity (Fig. 4).

Endometrial cancer is isointense to the endometrium on T1-weighted images and it most frequently shows heterogeneous moderate to high-signal intensity on T2-weighted images. However, it can also show low-signal intensity on T2-weighted images (Fig. 5).

On DCE-MR, in the arterial phase $(\approx 30 \mathrm{~s})$ endometrial tumors enhance earlier than does the

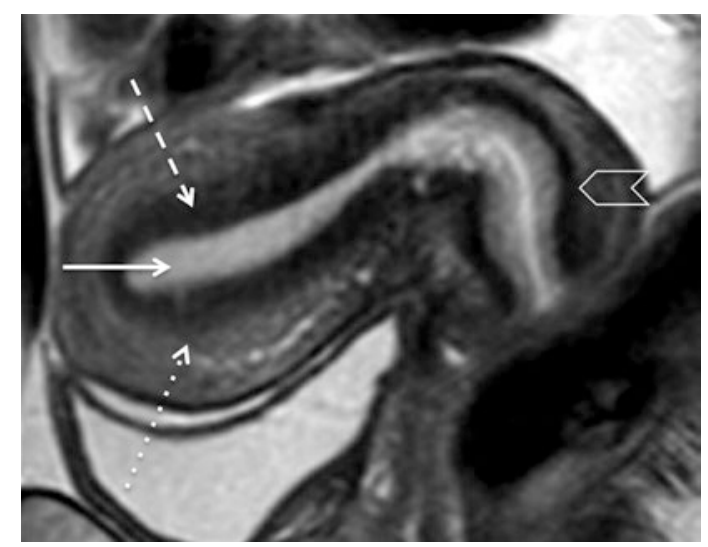

Fig. 4 Normal uterine T2-weighted anatomy. Sagittal T2-weighted image. Hyperintense endometrium (arrow); hypointense junctional zone (dashed arrow); outer myometrium showing intermediate intensity (dotted line); hypointense cervical stroma (arrowhead)

normal endometrium, as this is the most adequate sequence to depict small endometrial tumors confined to the endometrium. At the equilibrium phase ( $\approx 120-180$ s), tumors tend to be hypointense relative to the myometrium. However, they can remain isointense and a minority can even be hyperintense (Figs. 5 and 6).

On DWI-MR the tumors usually show restricted diffusion (hyperintensity on high b-value sequences and hypointensity on the respective ADC map) (Fig. 6). ADC values have shown to be significantly lower in endometrial carcinoma than in normal endometrium and benign conditions such as endometrial polyps and submucosal leiomyomas (Fujii et al. 2008; Tamai et al. 2007; Takeuchi et al. 2009; Rechichi et al. 2011) (Fig. 7).Therefore, this functional sequence can be helpful in diagnosing an endometrial tumor when biopsy cannot be easily performed (i.e., in the case of cervical stenosis) or when the histopathological diagnosis is not conclusive. Cross-reference with conventional sequences is, however, mandatory to avoid pitfalls, since restricted diffusion may be present also in retained secretions in the endometrial cavity.

Several studies have shown that there is no relation between the tumor grade, aggressiveness, and ADC values (Rechichi et al. 2011; 

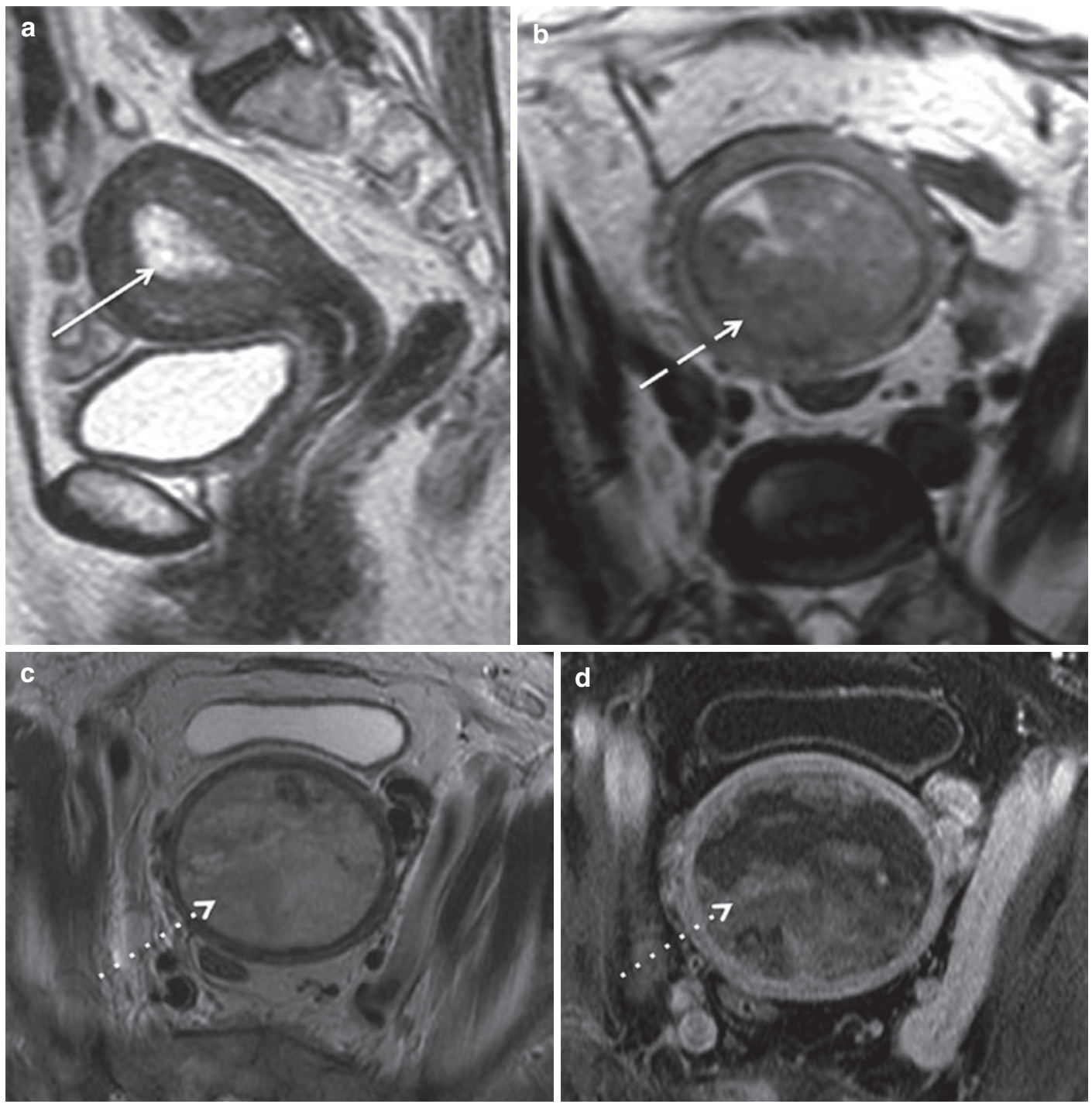

Fig. 5 MR features of endometrial cancer. (a) endometrioid cancer showing heterogeneous high-signal intensity on sagittal T2-weighted image (arrow); (b) endometrioid cancer showing hyposignal intensity on axial oblique T2-weighted image, a less common appearance (dashed arrow); (c, d) endometrioid cancer distending the uterine

Bharwani et al. 2011). However, in one study higher grade tumors were associated with lower ADC values but there was a considerable overlap in estimation of histological grade based on ADC values (Tamai et al. 2007). Moreover, high ADC values may be found in high-grade tumors with a large necrotic component (Sala et al. 2010). cavity and compressing the myometrium showing a remarkable heterogeneous high-signal intensity on axial oblique T2 weighted image (dotted arrows) and mixed hypointensity and isointensity relative to outer myometrium after administration of gadolinium in the equilibrium phase (dotted arrows)

\subsubsection{Stage I Disease}

Stage I endometrial carcinomas account for approximately $74 \%$ of endometrial cancers (Cancer Incidence Statistics 2015). The most important role of the radiologist, when facing a stage I endometrial cancer is to determine the extent of myometrial invasion, since stage I disease is subdivided into stage IA disease, if the 

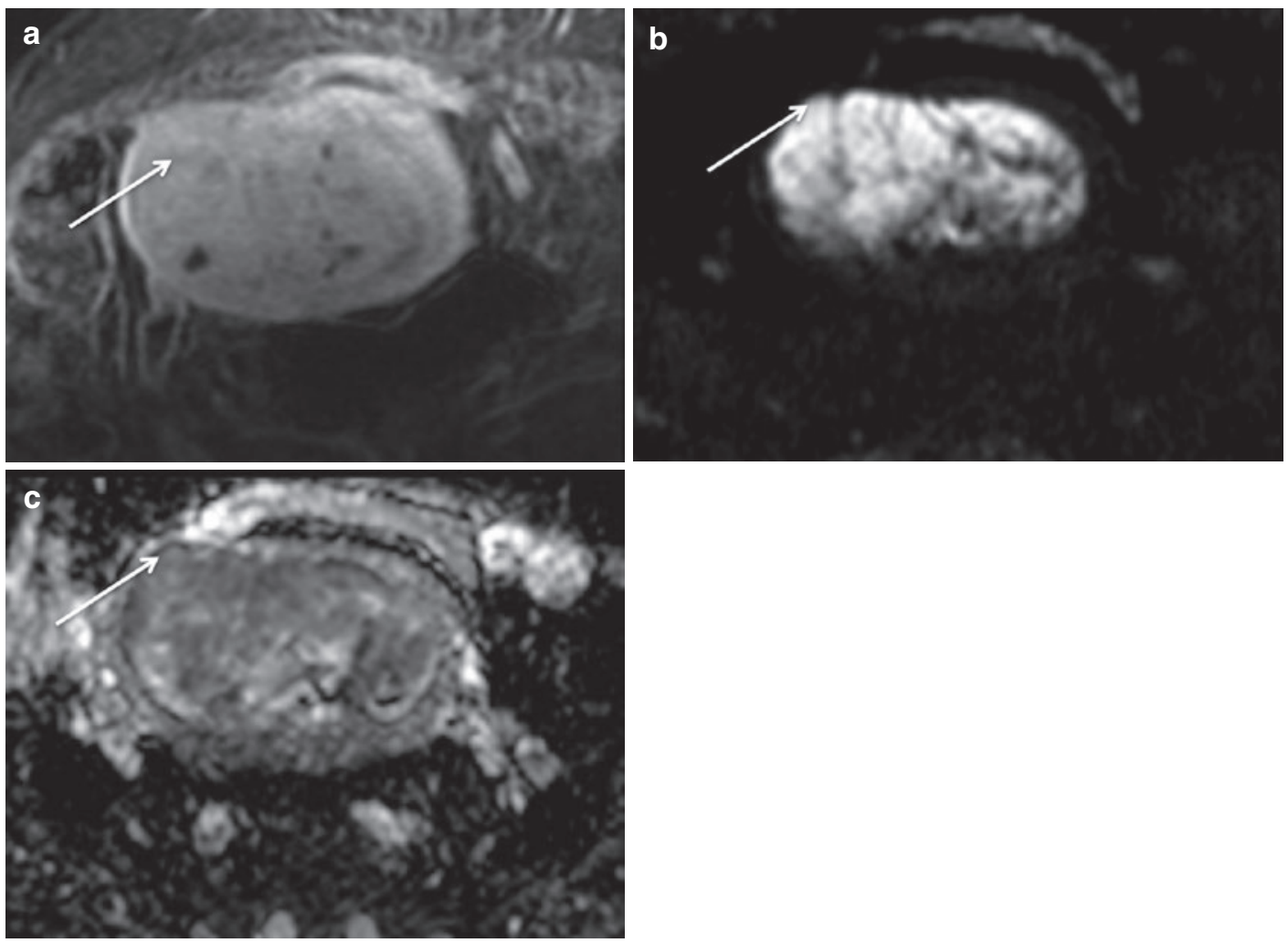

Fig. 6 (a) Endometrioid carcinoma in a 79-year-old woman, isointense to the myometrium after administration of gadolinium in the equilibrium phase, making it difficult to access the depth of myometrial invasion (arrow);

tumor only invades $<50 \%$ of the myometrium, and stage IB disease, if the tumor invades $\geq 50 \%$ of the myometrium (Figs. 8 and 9) (Pecorelli 2009). If the patient is considering fertility-sparing treatment, it is also important to clearly define if the tumor is confined to the endometrium or if it invades the superficial myometrium, which precludes this approach (Fig. 10) (Jafari Shobeiri et al. 2013; Kesterson and Fanning 2012).

$\mathrm{T} 2$-weighted images are useful in determining myometrial invasion. A focal or diffuse thickening of the endometrium with a clearly visualized intact junctional zone is a sign that the tumor is confined to the endometrium (Fig. 8). Breaching and irregularity of the hypointense signal of the junctional zone implies superficial myometrial invasion (Manfredi et al. 2005).

The depth of myometrial invasion should be based on the thickness of remaining myometrium

(b, c) diffusion-weighted image $\left(b=1000 \mathrm{~mm} / \mathrm{s}^{2}\right)$ and the respective ADC map show typical tumoral restricted diffusion, enabling the radiologist to diagnose invasion of $\geq 50 \%$ the myometrium (stage IB) (arrow)

where the tumor is at its deepest point into the myometrium (Haldorsen and Salvesen 2012; Koyama et al. 2007).

However, there are situations where the evaluation of myometrial invasion by $\mathrm{T} 2$-weighted images may be difficult or even impossible, such as the presence of: a tumor in the cornua (Fig. 11); a fibromatous uterus (Fig. 11); a less conspicuous junctional zone in postmenopausal women (Fig. 12); a thinned myometrium in postmenopausal women (Fig. 13); an adenomyotic uterus (Fig. 14); a polypoid uterus; a congenital abnormality (Fig. 15 ); and the presence of poor tumor signal difference/isointensity between the tumor and the myometrium (Figs. 6 and 16) (Kinkel 2006; Scoutt et al. 1995; Yamashita et al. 1993a; Saez et al. 2000; Fanning et al. 1990). In these cases, DCE-MR and DWI-MR play an important role, when combined with T2-weighted images. 

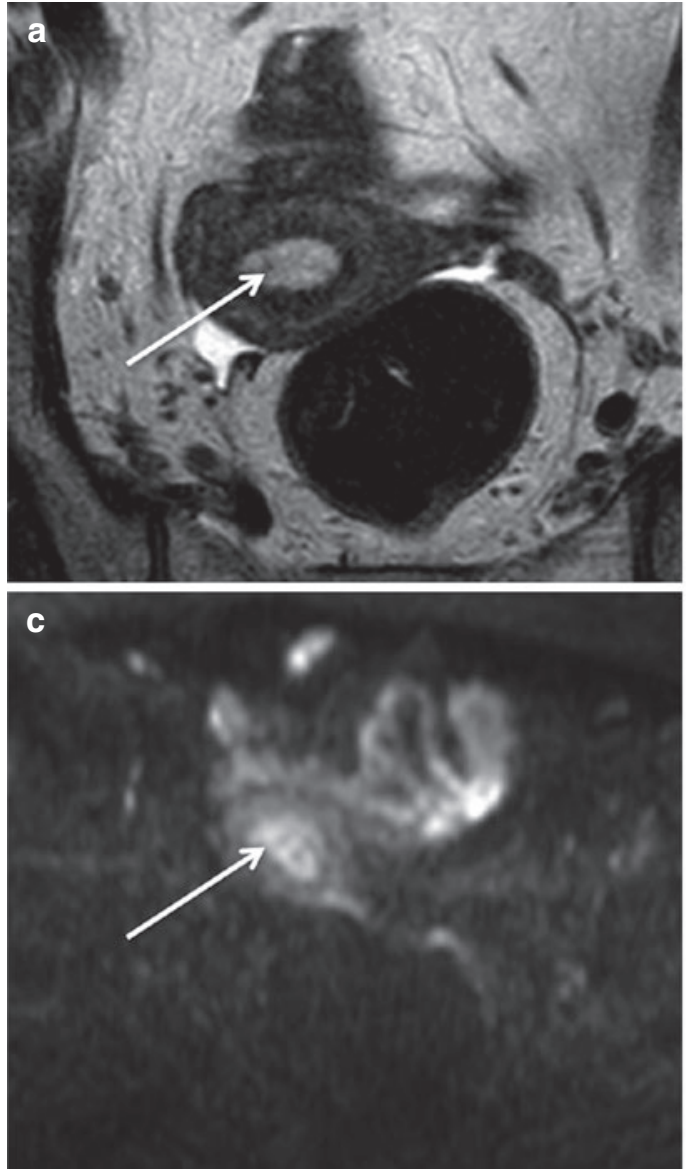

Fig. 7 Benign polyp in a 60-year-old woman. (a) Axial oblique T2-weighted image shows a heterogeneous hyperintense filling of the endometrial cavity; (b) axial oblique 3D fat-suppressed T1-weighted sequence after the administration of gadolinium in the arterial phase (30 s) shows lesional heterogeneous enhancement (arrow). Note the avid enhancement of the junctional zone in this phase when compared to the outer myometrium (dashed

On DCE-MR imaging, in the arterial phase $(\approx 30 \mathrm{~s})$, the junctional zone typically enhances avidly when compared to the endometrial tumor (Manfredi et al. 2005). Disruption of this subendometrial enhancement usually indicates myometrial invasion (Fujii et al. 2015; Manfredi et al. 2005; Nakao et al. 2006; Yamashita et al. 1993b; Kaneda et al. 2011; Frei and Kinker 2001). This is particularly important when the patient is being considered for fertility-sparing treatment since the FIGO 2009 division of stage I disease is only based on the presence of invasion of the inner or
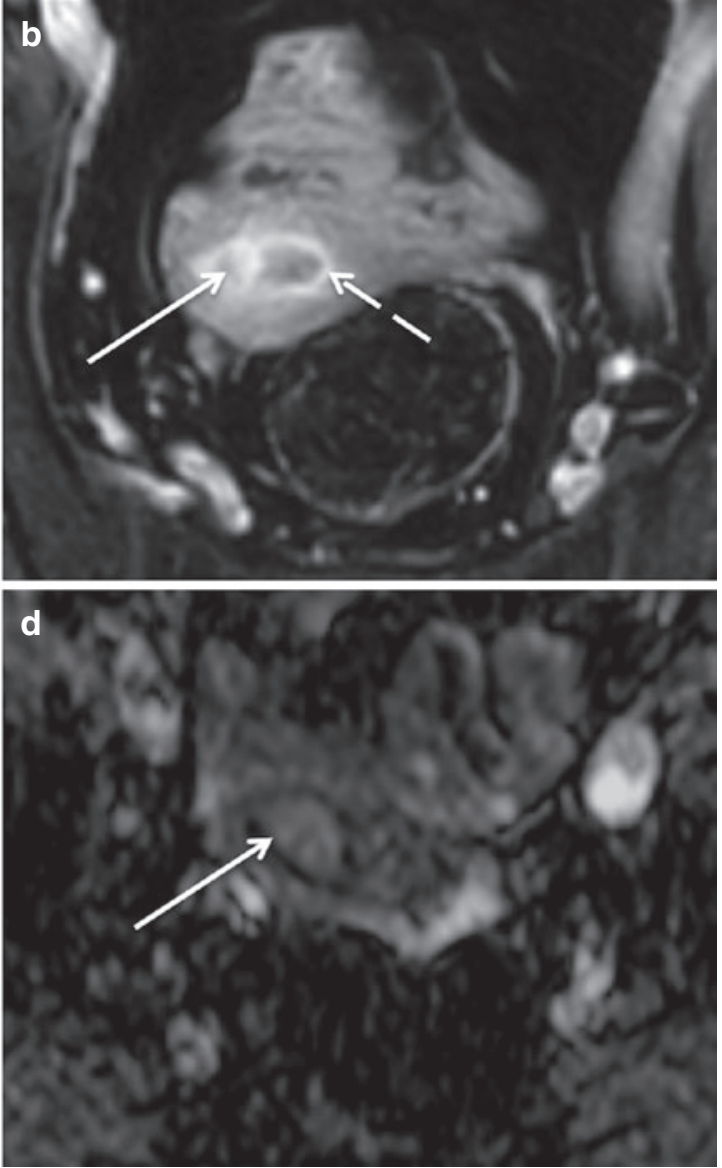

arrow). Both $\mathrm{T} 2$ and DCE-MR characteristics of this benign polyp mimic those of endometrial carcinoma; (c) axial diffusion-weighted image $\left(b=1000 \mathrm{~s} / \mathrm{mm}^{2}\right)$ shows high-signal intensity of the tumor; (d) the apparent diffusion coefficient map also shows high-signal intensity. In contrast to what would be expected in an endometrial carcinoma, this benign polyp does not show restricted diffusion

outer portions of the myometrium (Fig. 10) (Jafari Shobeiri et al. 2013; Kesterson and Fanning 2012).

On the other hand, it is in the equilibrium phase $(\approx 120 \mathrm{~s})$ that is better diagnosed deep myometrial invasion. In this phase there is more pronounced contrast-to-noise ratio between the outer myometrium, which is markedly hyperintense, and the endometrial tumor, which is usually hypointense (Figs. 9, 13 and 15) (Manfredi et al. 2005). Attention must be paid to the presence of peritumoral inflammatory enhancement, which 


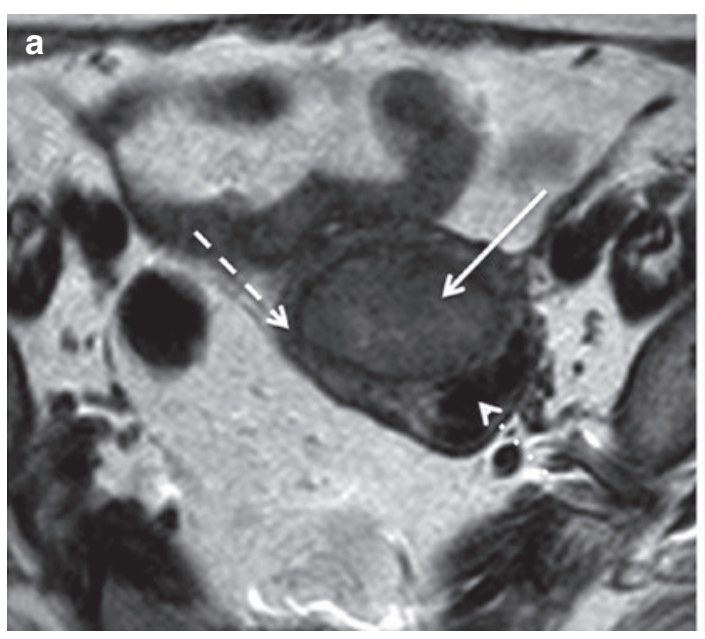

Fig. 8 Stage IA endometrioid carcinoma. (a) Axial oblique T2-weighted image; (b) axial oblique 3D fatsuppressed T1-weighted sequence after the administration of gadolinium in the arterial phase (30 s). There is preservation of the regular hypointense signal on $\mathrm{T} 2$-weighted

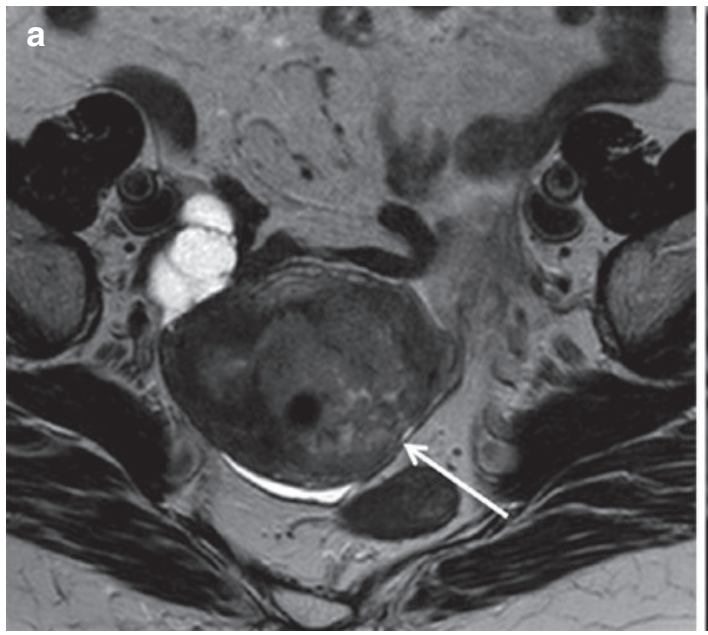

Fig. 9 Stage IB endometrioid carcinoma in an 85-yearold woman. (a) Axial oblique T2-weighted image; (b) axial oblique 3D fat-suppressed T1-weighted sequence after the administration of gadolinium in the equilibrium phase $(120 \mathrm{~s})$. There is extension of the tumor to the outer

can exaggerate the appearance of myometrial invasion (Sala et al. 2013).

DWI-MR has shown to be very useful in assessing myometrial invasion, especially in patients who cannot receive intravenous contrast, in patients with isointense or hyperintense tumors

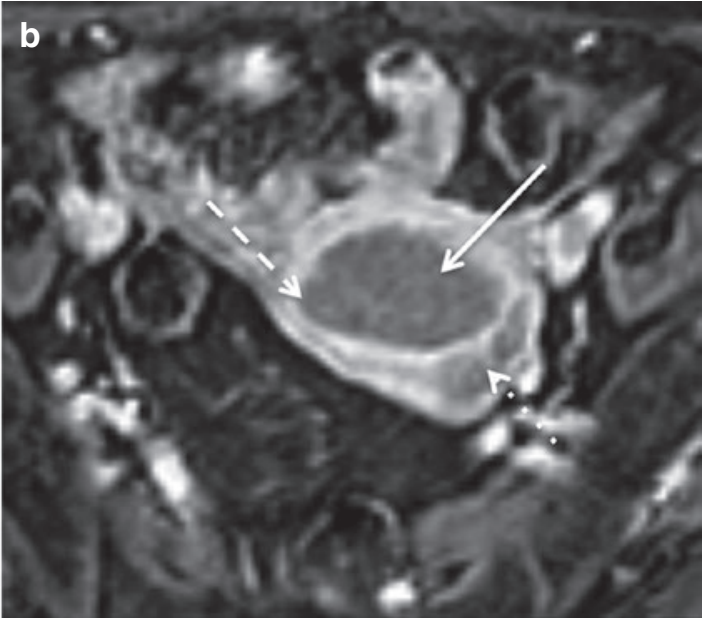

image and of the regular enhancement of the junctional zone after administration of gadolinium in the arterial phase (dashed arrow), thus the tumor is confined to the endometrial cavity (arrow). Note the intramural leiomyoma in the posterior corpus (dotted arrow)

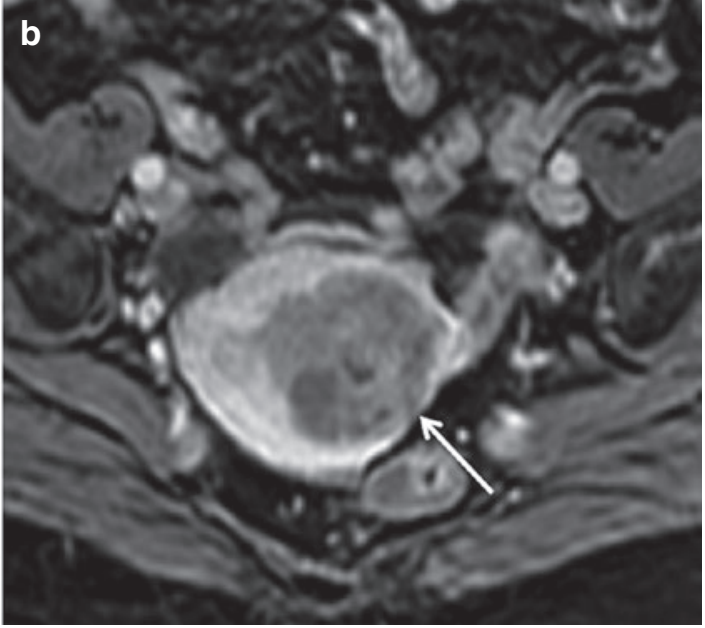

portion of the left posterior myometrium (arrow), which is clearly depicted after the administration of gadolinium in the equilibrium phase, where there is more pronounced contrast-to-noise ratio between the hypointense tumor and the hyperintense outer myometrium

relative to the myometrium following contrast administration, and in patients with adenomyosis (Figs. 16, 14 and 16) (Takeuchi et al. 2009; Sala et al. 2010).

Moreover, DWI-MR is very accurate in assessing myometrial invasion. 


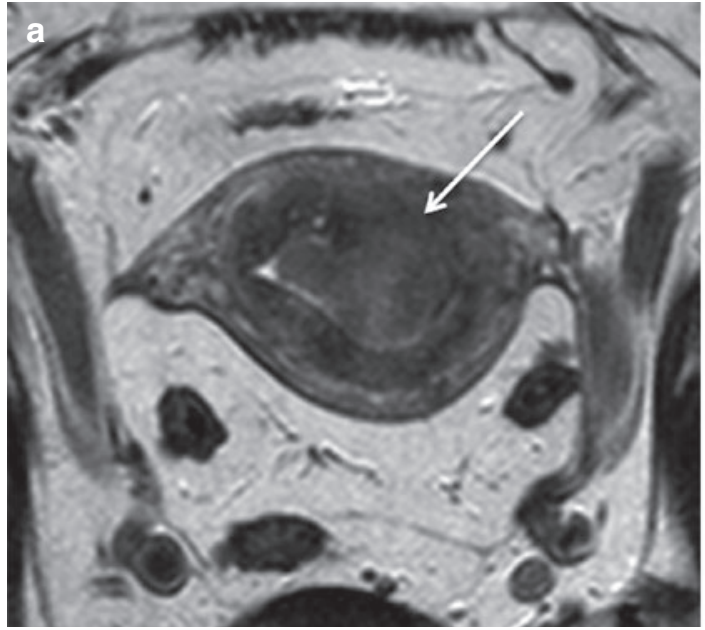

Fig. 10 Endometrioid carcinoma in a woman considering fertility-sparing therapy. (a) Axial oblique T2-weighted image; (b) axial oblique 3D fat-suppressed T1-weighted sequence after the administration of gadolinium in the arterial phase (30 s). Axial oblique T2-weighted image is

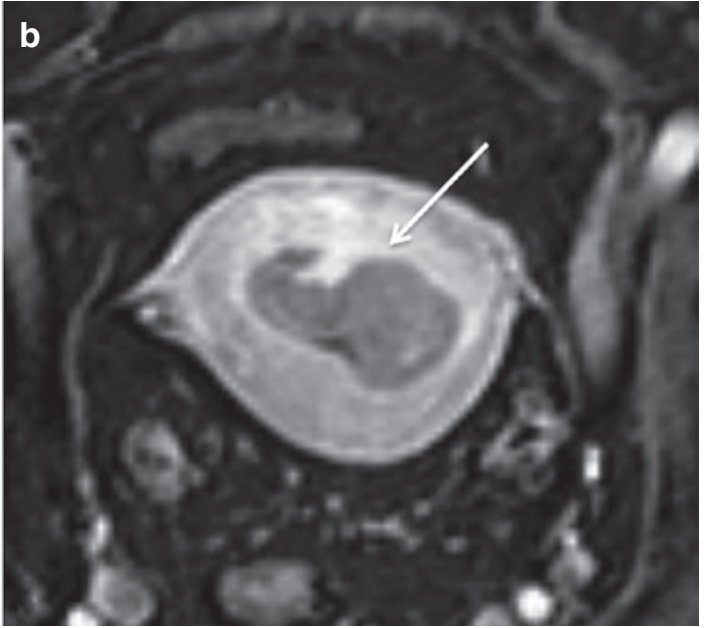

inconclusive regarding myometrial invasion, whereas DCE-MR in the arterial phase clearly shows that there is no disruption of the junctional zone enhancement and therefore the tumor does not invade the myometrium
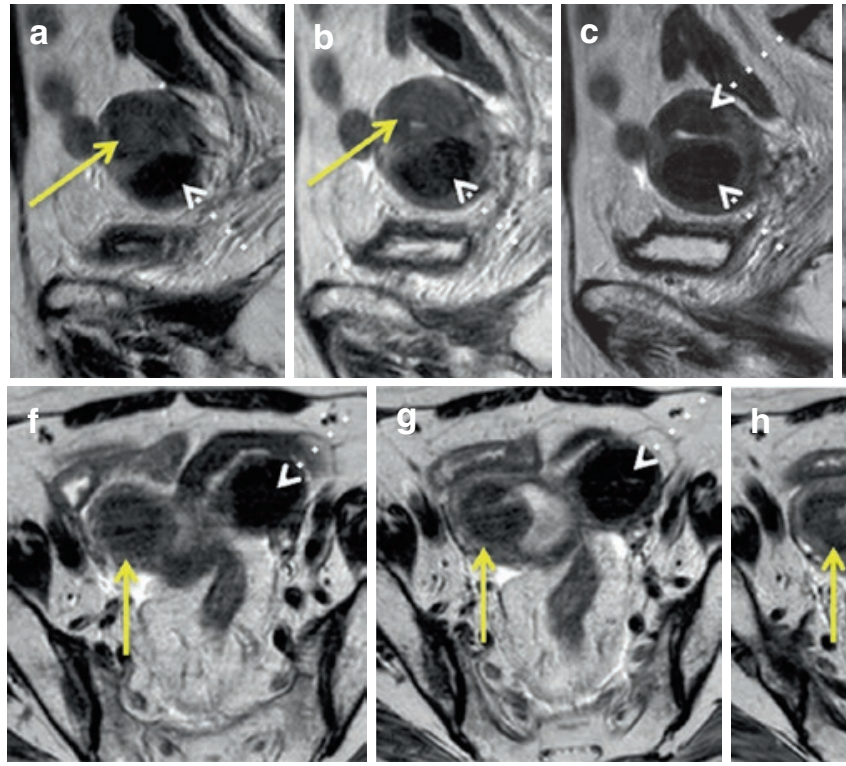
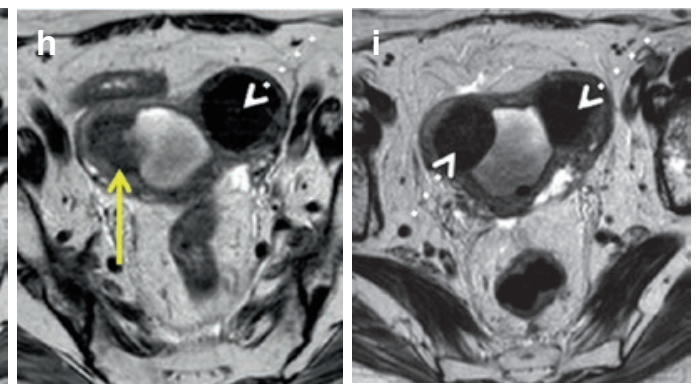
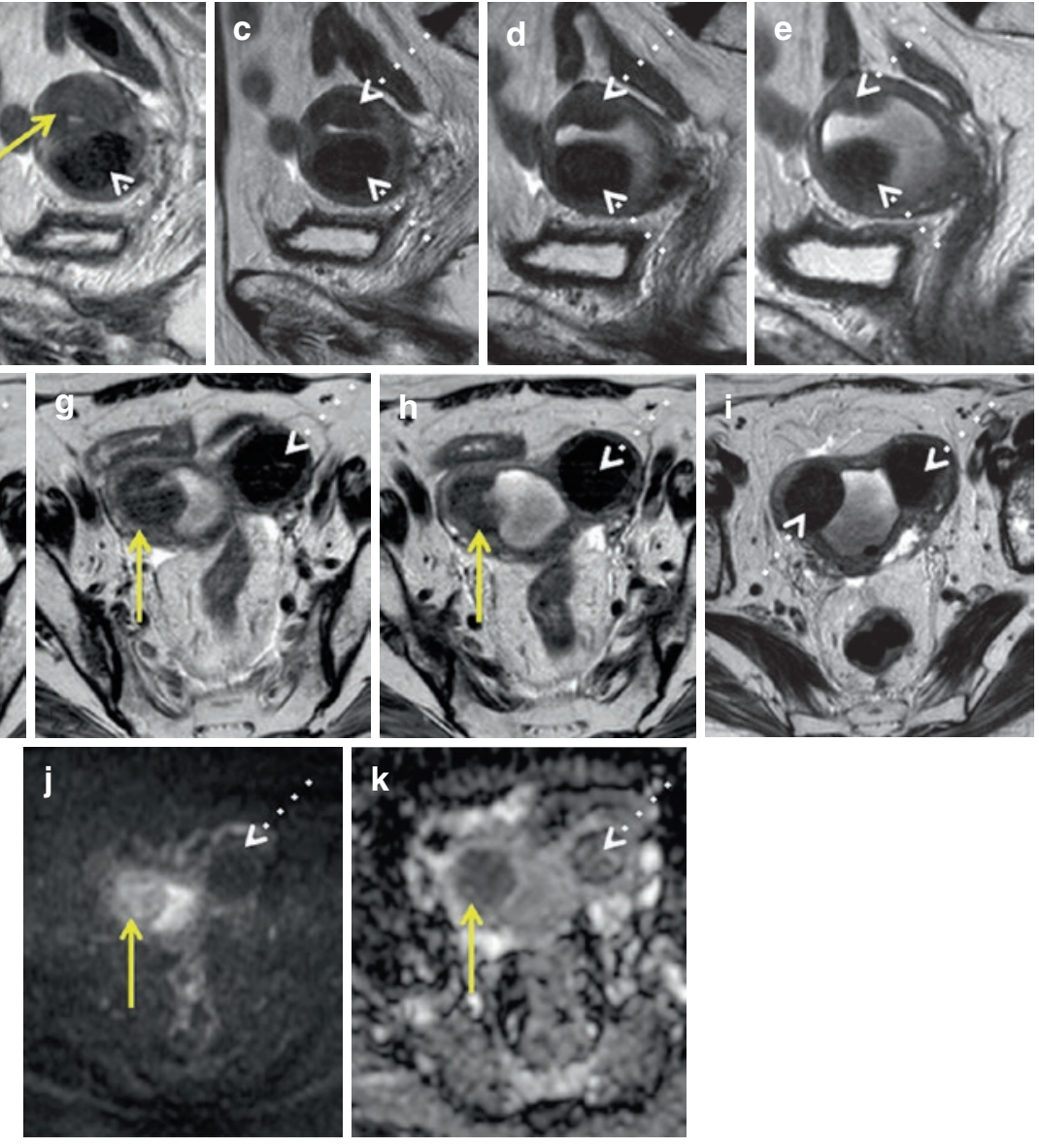

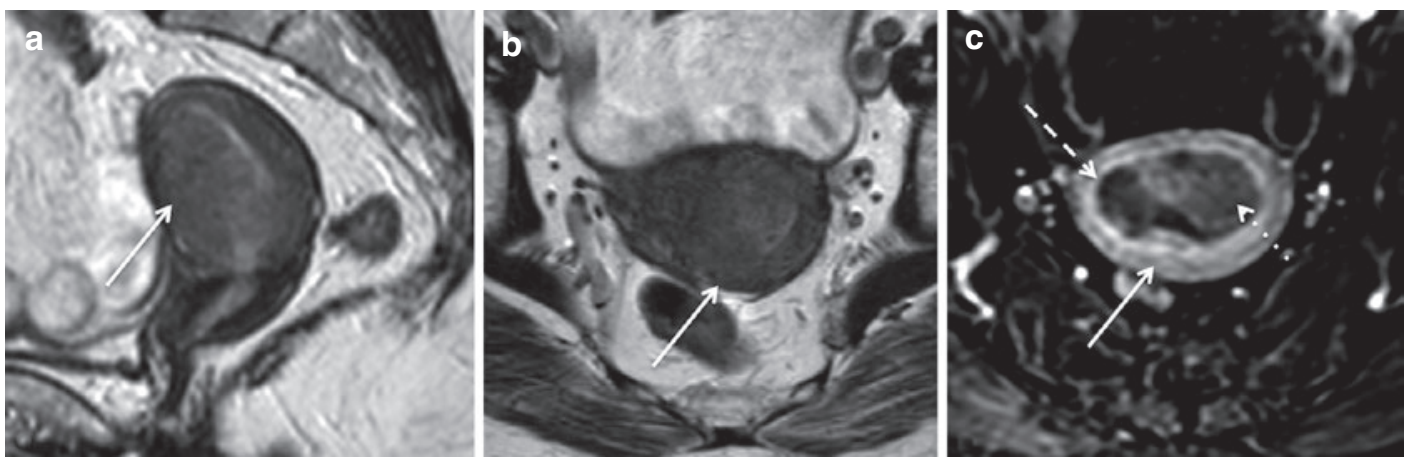

Fig. 12 Stage IA mucinous carcinoma of the endometrium in a 72-year-old woman with a thinned junctional zone. (a) Sagittal T2-weighted image; (b) axial oblique T2-weighted image; (c) axial oblique 3D fat-suppressed T1-weighted sequence after the administration of gadolinium in the arterial phase $(30 \mathrm{~s})$. The junctional zone of this postmenopausal woman cannot be identified in $\mathrm{T} 2$-weighted images, making it difficult to assess the depth of tumoral myometrial invasion. The use of T2-weighted images alone might suggest that the tumor invades the outer portion of the myometrium (arrow). However contrast-enhanced image in the arterial phase clearly shows an enhancing regular junctional zone (dashed arrow) and the tumor confined to the endometrial cavity (dotted arrow)
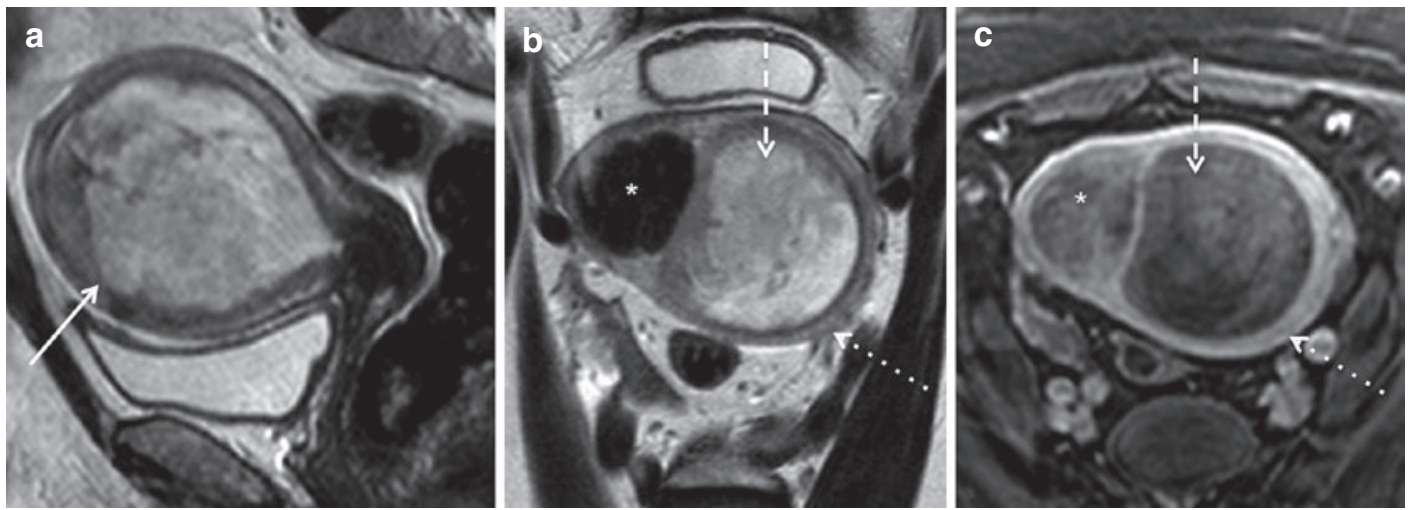

Fig. 13 Stage IA endometrioid carcinoma in a 72-year-old woman with myometrial thinning secondary to stretching due to a large tumor. (a) Sagittal T2-weighted image; (b) axial T2-weighted image; (c) axial oblique 3D fat-suppressed T1-weighted sequence after the administration of gadolinium in the equilibrium phase (120 s). Distortion at the base of this polypoid tumor is difficult to distinguish from outer myometrial tumoral invasion on T2 weighted images (arrow). Contrast-enhanced image in the equilibrium phase clearly shows a hypointense tumor (dashed arrow) that does not invade the outer hyperintense myometrium (dotted arrow). Note the T2 hypointense intramural leiomyoma (asterisk)
Fig. 11 Endometrial adenocarcinoma in a 71-year-old patient, previously submitted to radiation therapy due to cervical cancer. (a-e) Sagittal T2-weighted images fail to clearly show a small isointense tumor in the right uterine cornua (yellow arrow), due to the coexistence of submucosal leiomyomas (dotted arrow); (f-i) axial oblique $\mathrm{T} 2$-weighted images better depict this isointense lesion and clearly differentiate it from the hypointense leiomyomas; (j) axial diffusion-weighted image $\left(b=1000 \mathrm{~s} / \mathrm{mm}^{2}\right)$ shows high-signal intensity of the tumor; (k) the apparent diffusion coefficient map shows hyposignal intensity, therefore tumoral restricted diffusion. In contrast, the leiomyoma present in the left cornua, does not show restricted diffusion 


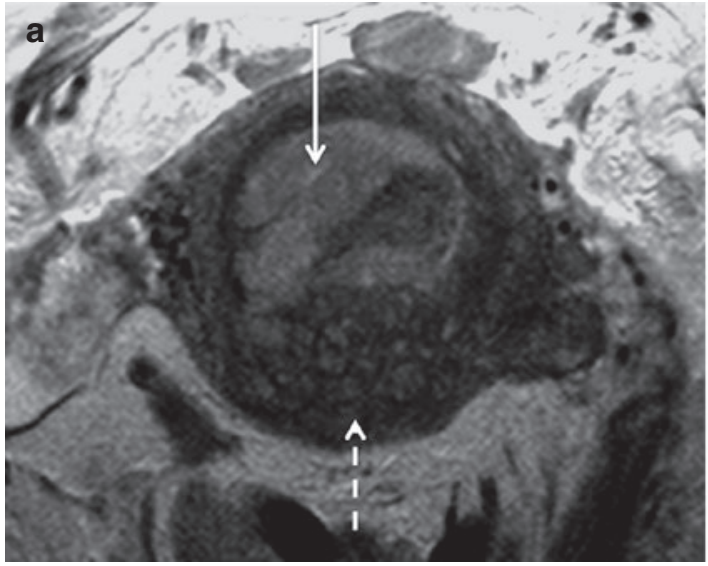

Fig. 14 Endometrioid carcinoma in a 71-year-old woman with an adenomyotic uterus. (a) Axial oblique T2-weighted image. (b) Axial oblique 3D fat-suppressed $\mathrm{T} 1$-weighted sequence after the administration of gadolinium in the equilibrium phase (120 s). The

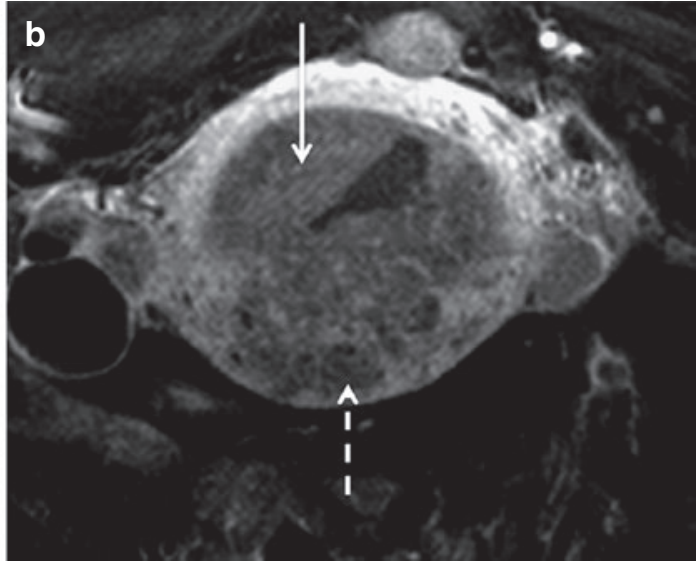

presence of posterior adenomyosis (dashed arrow) impedes the assessment of tumoral (arrow) myometrial invasion on both T2-weighted image and contrastenhanced image. Diffusion-weighted images could be useful in this case
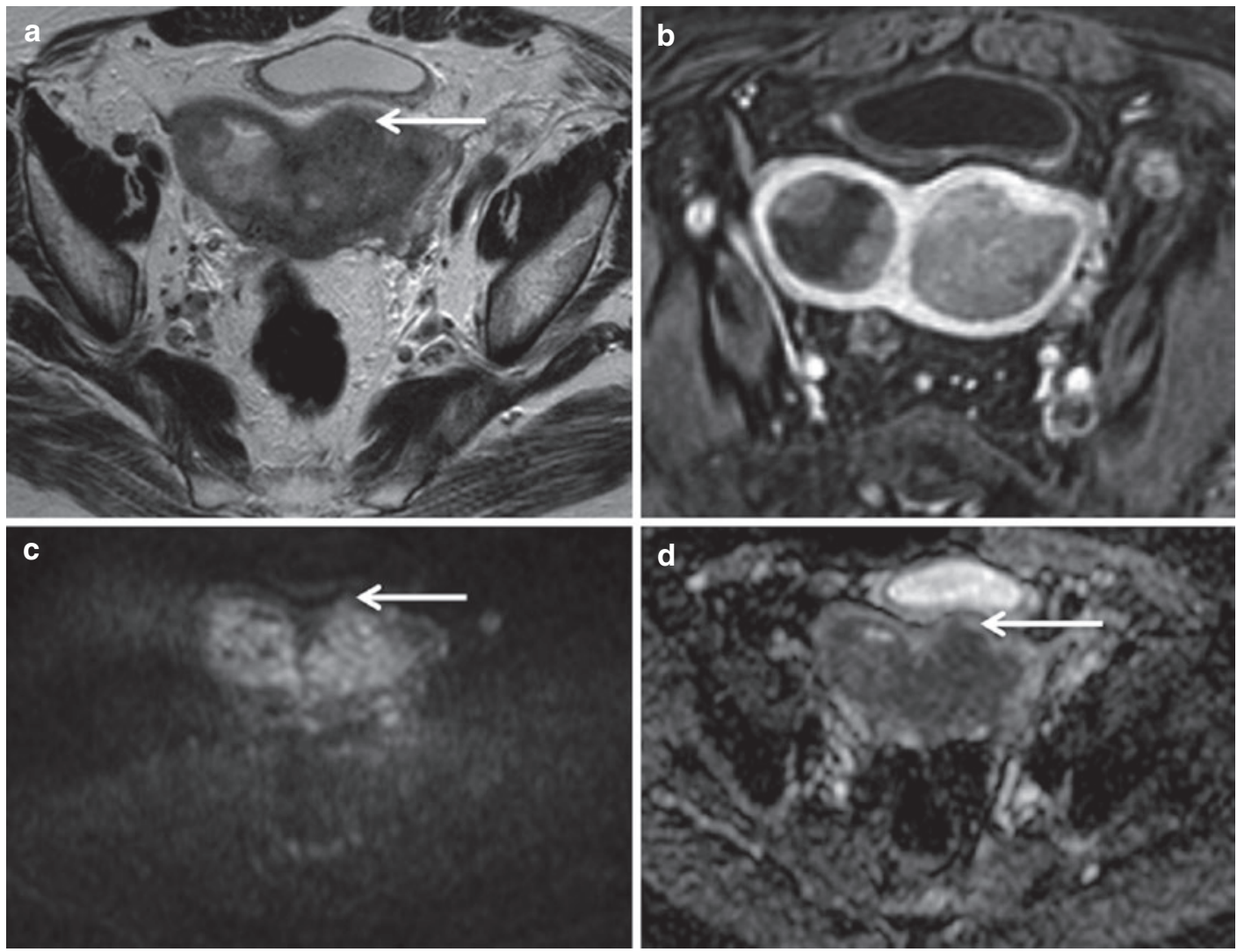

Fig. 15 Stage IB endometrioid carcinoma in a 76-yearold woman with a bicornuate bicollis uterus. (a) Axial T2-weighted image; (b) axial oblique 3D fat-suppressed T1-weighted sequence after the administration of gadolinium in the equilibrium phase (120 s); (c) axial diffusionweighted image $\left(b=1000 \mathrm{~s} / \mathrm{mm}^{2}\right)$. (d) ADC map. In this bicornuate bicollis uterus, the tumoral invasion of the anterior outer myometrium is difficult to depict on both T2-weighted image and after administration of contrast. Diffusion-weighted image and the respective ADC map clearly identify a focal area of restricted diffusion in the anterior outer myometrium, staging the tumor as IB 

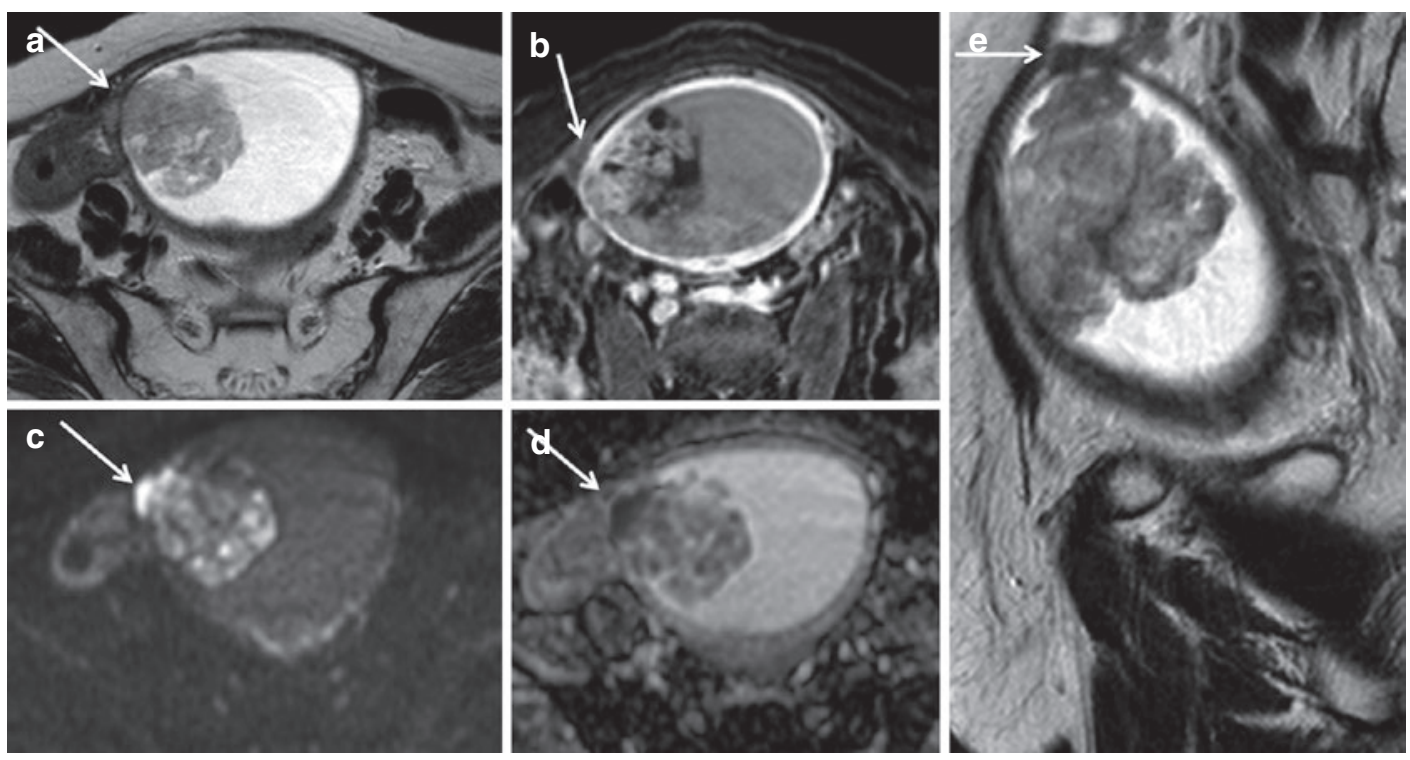

Fig. 16 Stage IB polypoid endometrioid carcinoma that distends the uterine cavity and causes myometrial thinning in a 68 year-old woman. (a) Axial T2-weighted image; (b) axial oblique 3D fat-suppressed T1-weighted sequence after the administration of gadolinium in the equilibrium phase (120 s); (c) axial diffusion-weighted image ( $b=1000 \mathrm{~s} /$ $\mathrm{mm}^{2}$ ); (d) ADC map; (e) sagittal T2-weighted image. The

Recent studies have shown that DWI-MR is significantly superior in the assessment of myometrial invasion when compared to DCE-MR (Bonatti et al. 2015; Rechichi et al. 2010; Takeuchi et al. 2009; Beddy et al. 2012).

A prospective study conducted by Rechichi et al. has concluded that DWI-MR is highly accurate in assessing the depth of myometrial invasion and perhaps it could replace DCE-MR as a complement to T2-weighted images in the preoperative evaluation of endometrial carcinoma (Rechichi et al. 2010). These findings were also supported by the study of Bonatti et al. that also stated that T2-weighted images and DWI could replace the combination of T2-weighted images and contrast-enhanced T1-weighted images in the preoperative staging of endometrial carcinoma (Bonatti et al. 2015).

Takeuchi and coworkers also reported an accuracy of $94 \%$ for DWI and of $88 \%$ for DCE-MR in the assessment of myometrial invasion (Takeuchi et al. 2009).

ADC maps have also shown to provide accurate measurement of the depth of myometrial invasion (Gallego et al. 2014). endometrial tumor is heterogeneous and shows both areas of hypointense and hyperintense signal relatively to the outer myometrium after administration of gadolinium in the equilibrium phase. This makes it difficult to assess myometrial invasion. Outer tumoral myometrial invasion in this thinned myometrium is better depicted in the diffusion-weighted image and in the respective ADC map (arrows)

Nonetheless, a recent meta-analysis assessing only nine studies showed that although DWI-MR has a slightly higher specificity in the diagnosis of myometrial invasion, DCE-MR and DWI-MR do not differ significantly in sensitivity and specificity in presurgical diagnosis of myometrial invasion (Andreano et al. 2014).

\subsubsection{Stage II Disease}

Stage II disease is characterized by cervical stromal invasion (Pecorelli 2009).

In the majority of cases, T2-weighted images are helpful in diagnosing cervical stromal invasion by depicting a heterogeneous hyperintense tumor disrupting the hypointense cervical stroma (Manfredi et al. 2005).

Axial oblique plane of the cervix (perpendicular to the cervical canal) postcontrast injection 3D-reccaled echo fat-suppressed T1-weighted images in a late phase $(\approx 80-$ $240 \mathrm{~s}$ ) are also very useful in assessing cervical stromal invasion, as they show the disruption of cervical enhancement by the tumor (Fig. 17) (Tirumani et al. 2013; Haldorsen and Salvesen 


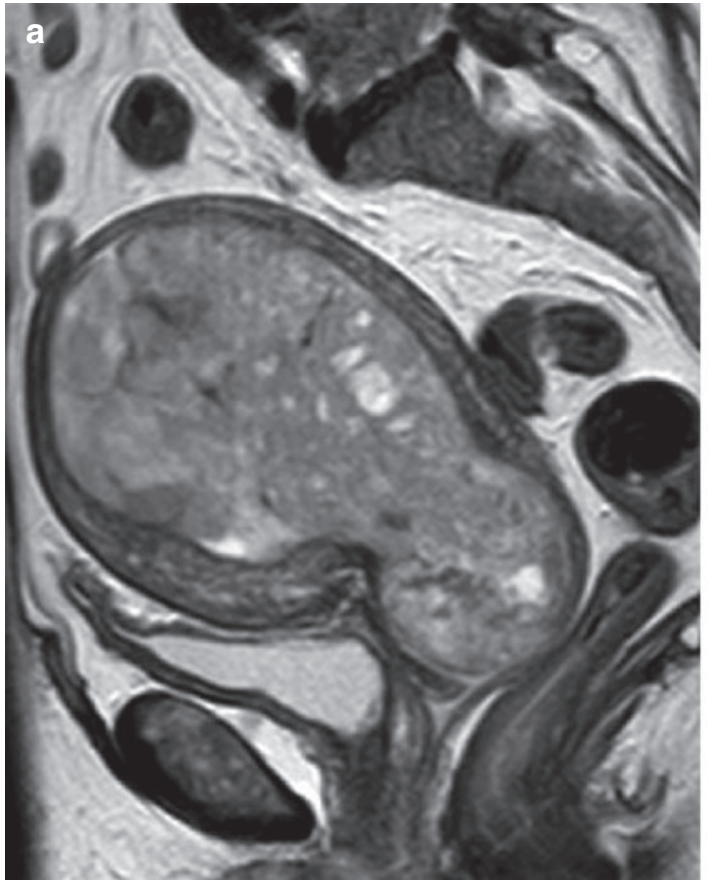

Fig. 17 Stage II endometrioid carcinoma in an 83-yearold woman (a) sagittal T2-weighted image; (b) axial oblique plane of the cervix (perpendicular to the cervical canal) postcontrast injection 3D-reccaled echo fatsuppressed T1-weighted images in a late phase (4 min).

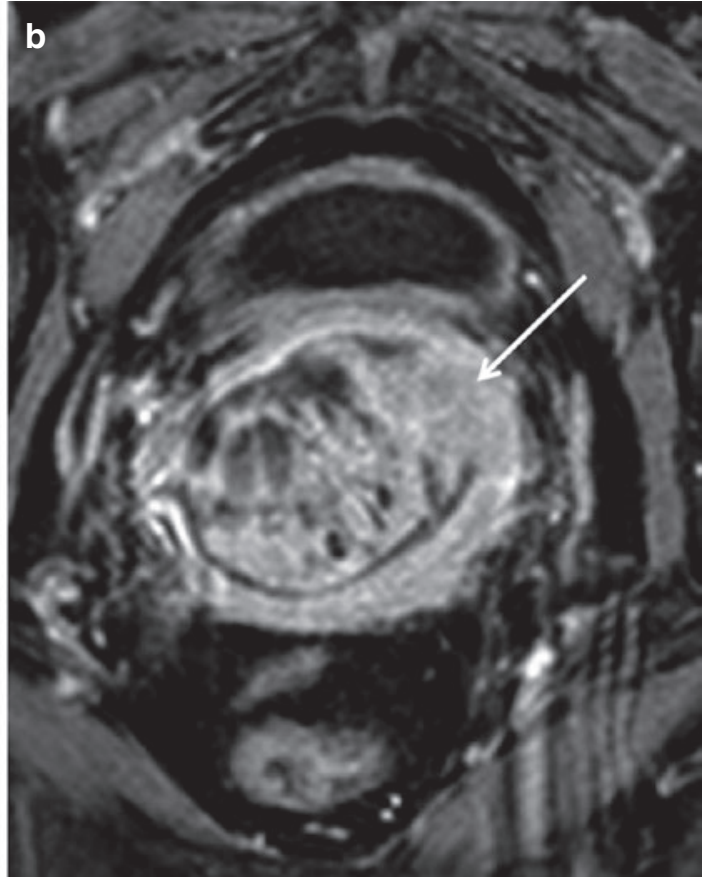

Sagittal T2-weighted image shows a voluminous endometrial tumor that prolapses in the cervical canal. The axial oblique plane of the cervix is very useful in detecting stromal invasion, especially after the administration of gadolinium in a late phase (4 min) (arrow)
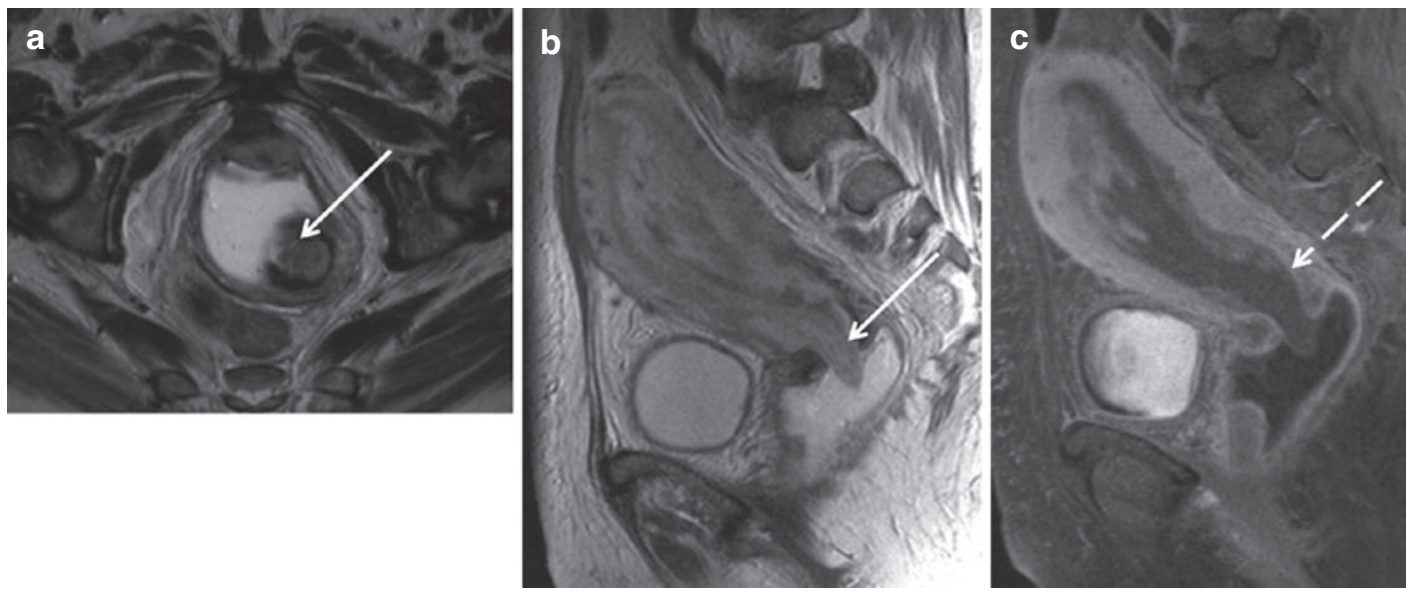

Fig. 18 Endometrial cancer prolapsed into the endocervical canal (a) Axial oblique plane of the cervix (perpendicular to the cervical canal) T2-weighted image: (b) sagittal T2-weighted image; (c) sagittal postcontrast injection 3D-reccaled echo fat-suppressed T1-weighted

2012; Seki et al. 2000). Normal enhancement of the cervical epithelium is present in tumors that project into the endocervical canal images in the equilibrium phase (120 s). Both axial oblique and sagittal T2-weighted images raise the suspicion of cervical invasion. However, there is normal enhancement of the cervical epithelium without any disruption, which excludes stage II disease

but do not invade the cervical stroma (Fig. 18) (Tirumani et al. 2013; Haldorsen and Salvesen 2012). 


\subsubsection{Stage III Disease}

Stage III disease is defined by local or regional spread of the tumor and it is subdivided into three substages (Pecorelli 2009).

In stage IIIA endometrial cancer, the tumor invades the serosa or/and the adnexa (Figs. 19 and 20).

Disruption of the serosal hypointense signal on T2-weighted images or loss of the enhancing myometrial outer margin on DCE-MR indicates serosal invasion (Fig. 19) (Tirumani et al. 2013; Manfredi et al. 2005).

The invasion of the ovaries may be due to direct extension via transmyometrial invasion or due to metastasis. Nodular foci in the adnexa are particularly frequent in type II endometrial can-

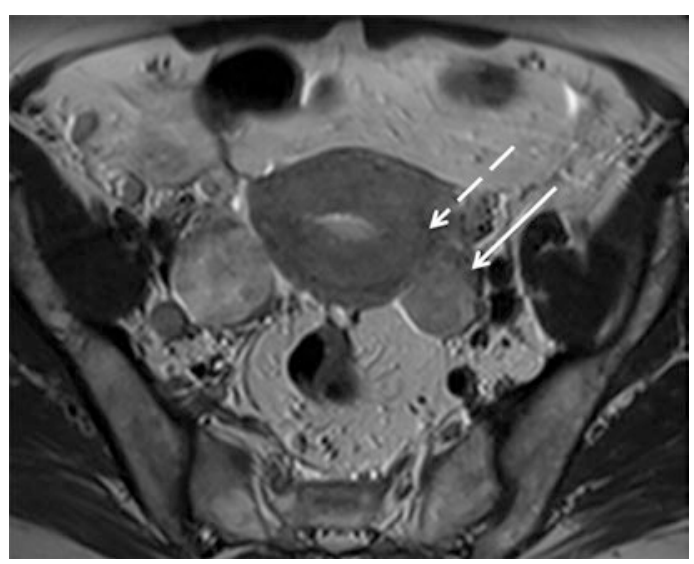

Fig. 19 Stage IIIA endometrioid carcinoma. (a) Axial oblique T2-weighted image shows disruption of the T2 hypointense serosal signal (dashed arrow) and tumoral invasion of the left ovary (arrow) cer and in high-grade endometrioid carcinoma (Tirumani et al. 2013). DWI-MR is very useful in depicting these cases as well as pelvic peritoneal disease (Fig. 20) (Tirumani et al. 2013; Sala et al. 2010; Shen et al. 2008).

In stage IIIB disease, there is tumoral involvement of the parametria and/or the vagina by direct extension or by skip metastasis (Figs. 21, 22 and 23) (Pecorelli 2009). Images of the axial oblique plane of the cervix (perpendicular to the cervical canal) are very helpful in detecting parametrial invasion (Fig. 21). Detection of parametrial invasion is particularly important so surgeons can avoid perform a simple hysterectomy and thus perform a more extensive resection (Sala et al. 2013).

In stage IIIC endometrial cancer there is lymph node involvement $\left(\mathrm{III}_{\mathrm{Cl}}\right.$ if there are positive pelvic lymph nodes and $\mathrm{III}_{\mathrm{C} 2}$ if there are positive paraaortic lymph nodes) (Fig. 24) (Pecorelli 2009).

MR sensitivity for detecting tumoral lymph node involvement is mostly low (Haldorsen and Salvesen 2012). The assessment of lymphadenopathies on MR imaging is still based on morphologic criteria (shortest axis diameter $>10 \mathrm{~mm}$ ) (Haldorsen and Salvesen 2012; Manfredi et al. 2004). DWI-MR helps detect lymph nodes, but there is a significant overlap with the ADC values of benign and malignant nodes (Nougaret et al. 2013; Nakai et al. 2008; Roy et al. 2010).

\subsubsection{Stage IV Disease}

Stage IV endometrial cancers are subdivided in two substages: stage IVA if there is invasion of
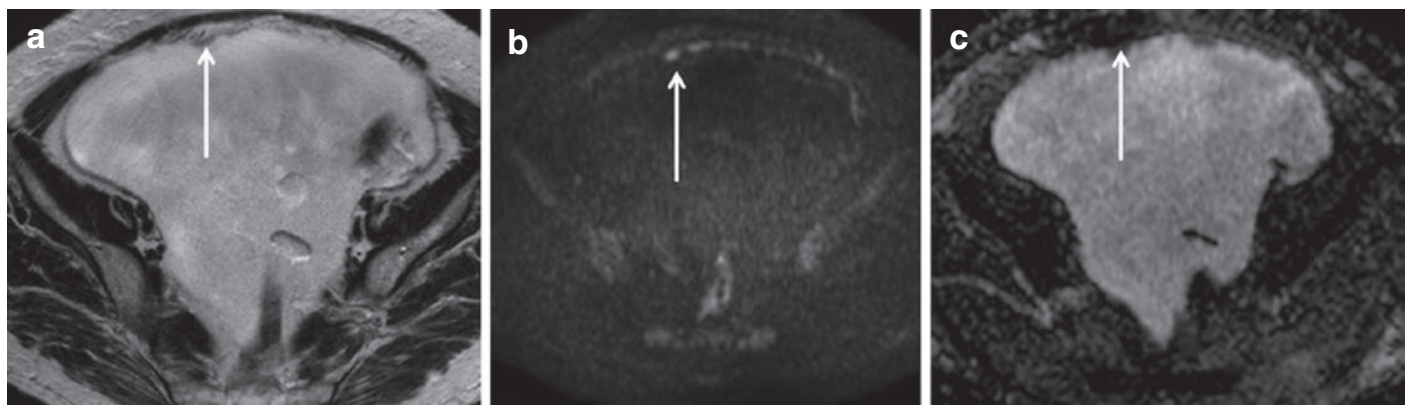

Fig. 20 Stage IIIA endometrioid carcinoma with subtle peritoneal nodules (a) axial T2-weighted image; (b) axial diffusion-weighted image $\left(b=1000 \mathrm{~s} / \mathrm{mm}^{2}\right) ;($ c) ADC map. Subtle peritoneal implants can be better depicted with diffusion-weighted images (arrow) 


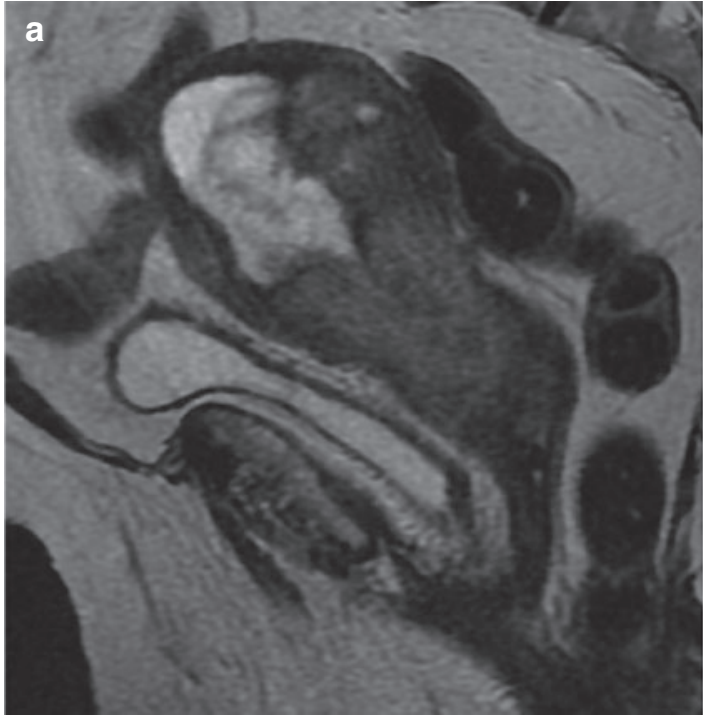

Fig. 21 Stage IIIB endometrioid carcinoma (a) sagittal T2-weighted image; (b) axial oblique plane of the cervix (perpendicular to the cervical canal) T2-weighted image. Parametrial invasion can be better depicted in the axial

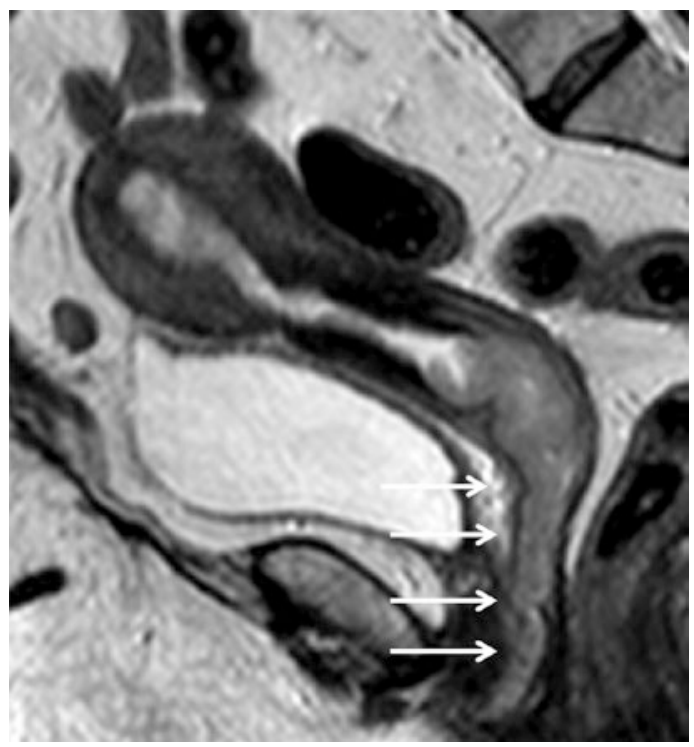

Fig. 22 Stage IIIB endometrioid carcinoma. (a) Sagittal T2-weighted image shows disruption of the T2 hypointense vaginal signal with invasion of the inferior third of the vagina (arrows)

the bladder or/and the bowel (Figs. 25 and 26); stage IVB if there are distant metastasis including abdominal metastasis and/or the presence of

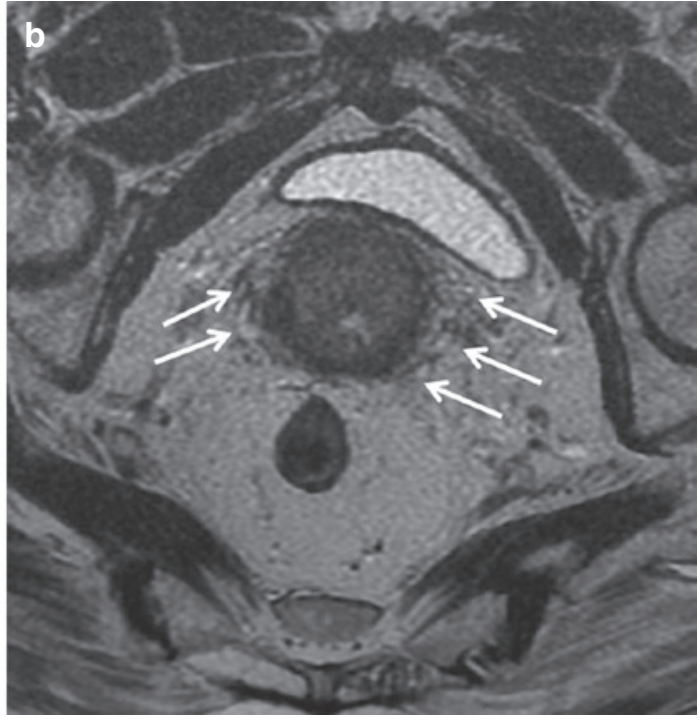

oblique plane of the cervix, where disruption of the hypointense stromal signal can be seen as well as tumor extending to both parametria (arrows)

positive inguinal lymph nodes or/and para-aortic nodes above the renal hila (Fig. 27) (Pecorelli 2009).

For an endometrial tumor to be considered as stage IVA, a lesion transgressing the hypointense T2-signal of the muscularis propria of the bladder and/or of the bowel must be clearly seen, as well as disrupting the hyperintense signal of the mucosa (Figs. 25 and 26) (Tirumani et al. 2013). Moreover, tumoral enhancement within the lumen should also be present (Figs. 25 and 26). This is particularly important in the differential diagnosis of bladder bullous edema (a sign of tumoral involvement of the bladder serosa and muscular layer, but not of the mucosa), since it is not considered stage IVA disease (Sala et al. 2013).

Although rare at presentation, distant hematogenous disease most commonly affects the liver, lungs, and bone (Fig. 27) (Numazaki et al. 2009).

In type 2 endometrial cancer, and in highgrade endometrioid endometrial cancer, stage IVB disease may present as in ovarian cancers with peritoneal carcinomatosis (Tirumani et al. 2013; Sala et al. 2013). 

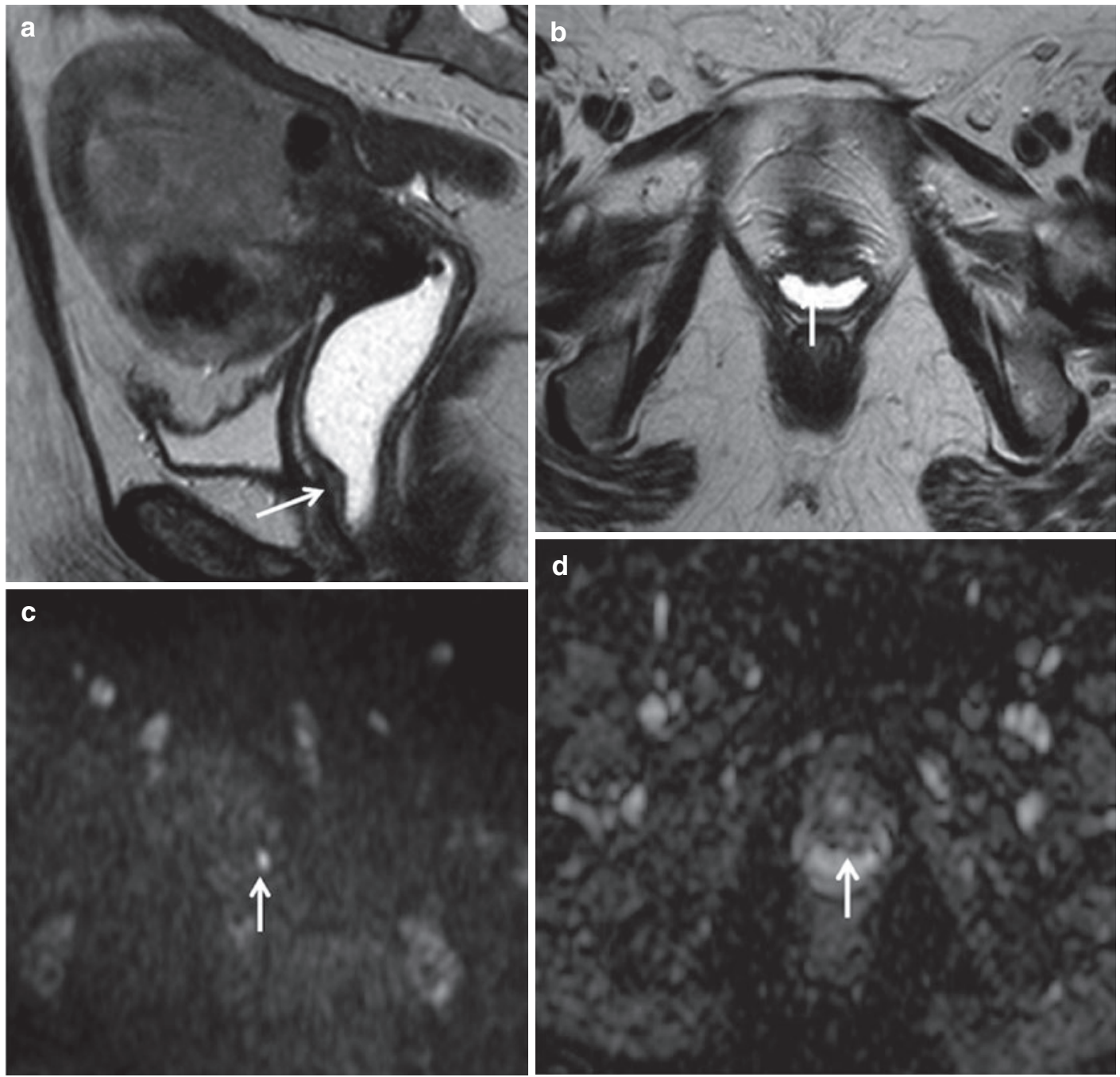

Fig. 23 Stage IIIB endometrioid carcinoma in a 62-yearold woman with skip vaginal metastasis. (a) Sagittal T2-weighted image; (b) axial T2-weighted image; (c) axial diffusion-weighted image $\left(b=1000 \mathrm{~s} / \mathrm{mm}^{2}\right) ;(d)$

ADC map. In the anterior lower third of the vagina a small lesion showing restricted diffusion can be seen (arrow). It was diagnosed as a vaginal metastasis

\section{Recent Advances in Functional MR Imaging in Assessing Endometrial Carcinoma}

In perfusion MR, repeated acquisitions of sequential images during the passage of gadolinium through the tumor are performed. Tumoral signal intensity versus time graphs and quantitative perfusion maps may be obtained, making it possible to evaluate microvascularity parameters

(Haldorsen and Salvesen 2012). The value of perfusion MR in assessing endometrial cancer is not yet established and validation studies are necessary to study its potential benefit.

Haldorsen et al. suggested that DCE-MRI may offer new information about the histological subtype and clinical course of endometrial carcinoma (Haldorsen et al. 2013). On the other hand, other studies regarding DCE-perfusion MR and tumor grading have shown contradictory results (Haldorsen et al. 2013; Ippolito et al. 2014a, b). 


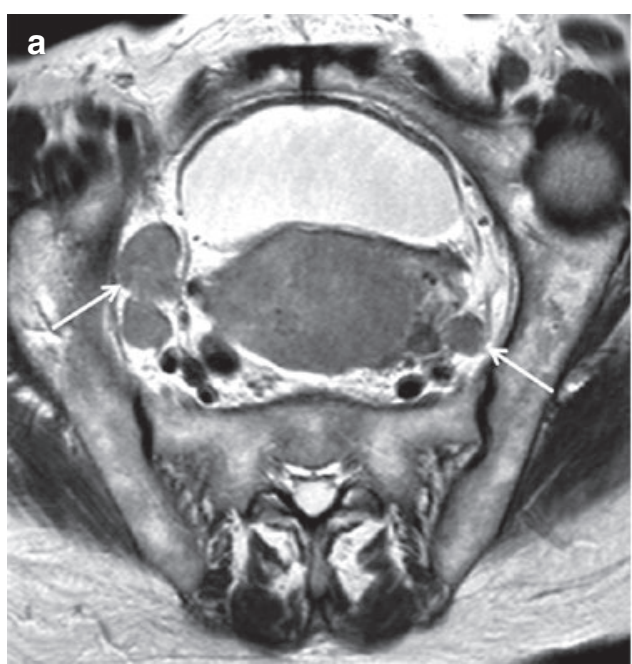

Fig. 24 Stage IIIC endometrial cancer (a) axial T2-weighted image shows pelvic lymphadenopathies (arrows) (stage $\mathrm{III}_{\mathrm{C} 1}$ disease); (b) axial $\mathrm{T} 2$-weighted

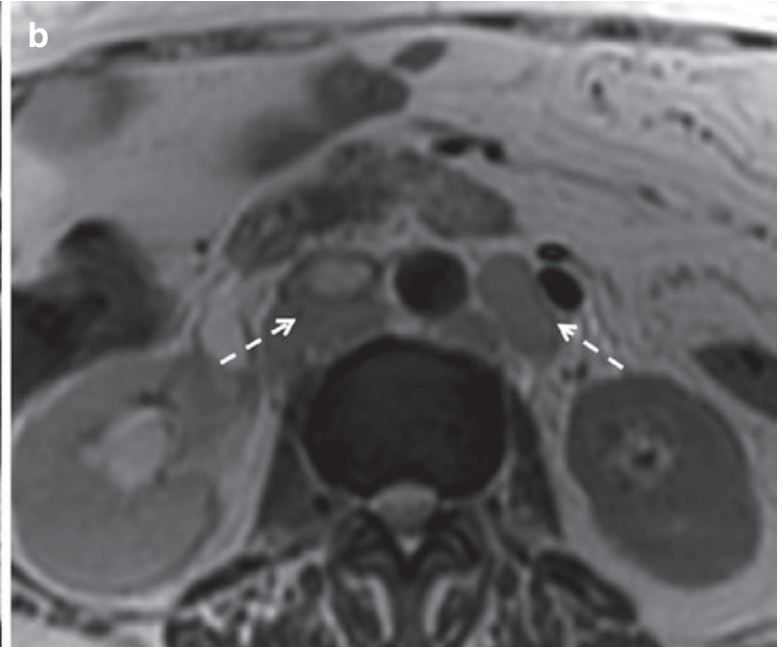

image shows para-aortic lymphadenopathies (dashed arrows) (Stage $\mathrm{III}_{\mathrm{C} 2}$ disease)
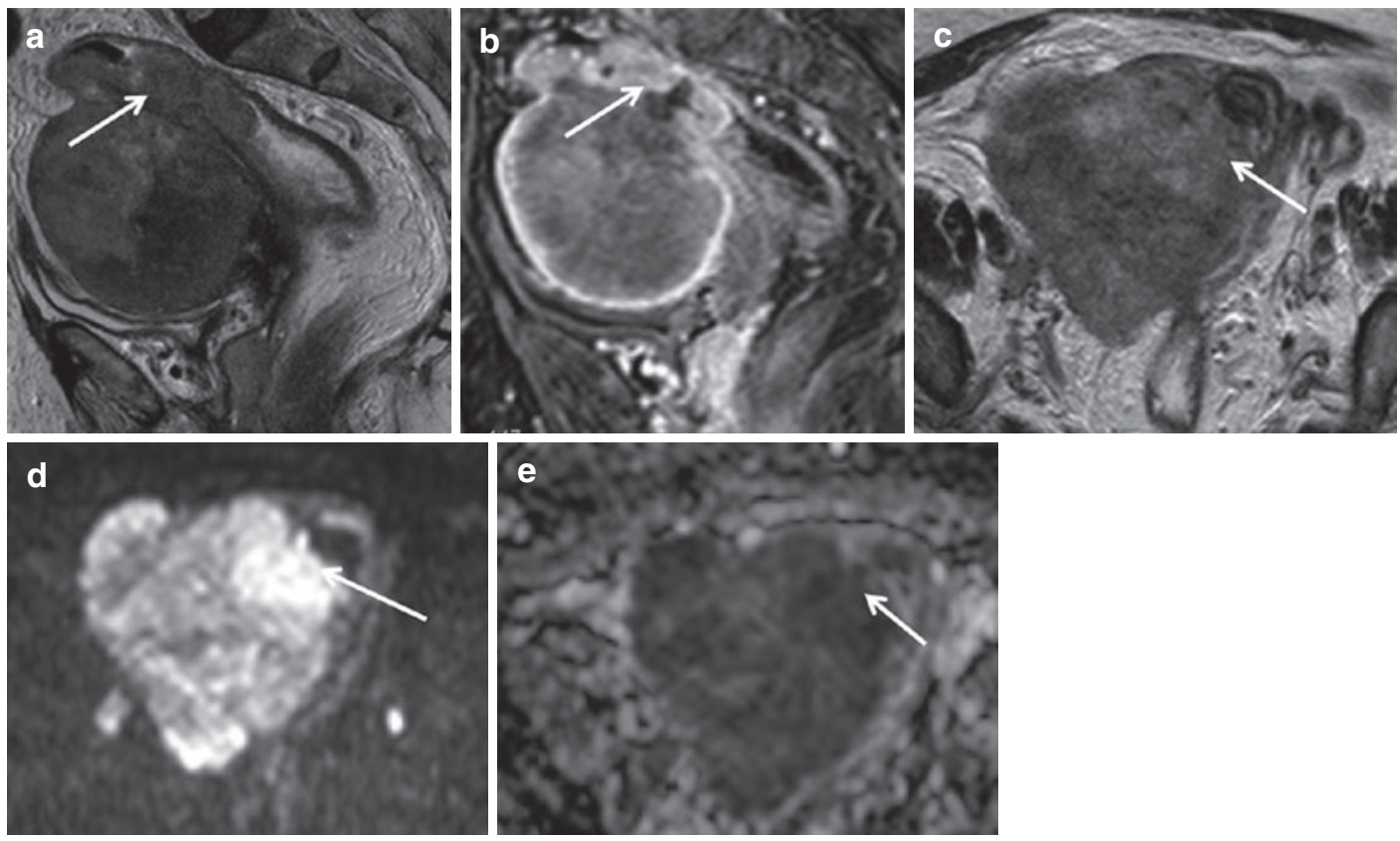

Fig. 25 Stage IVA endometrioid carcinoma that invades the sigmoid colon in a 75-year-old woman. (a) Sagittal T2-weighted image; (b) sagittal 3D fat-suppressed T1-weighted sequence after the administration of gadolinium in the equilibrium phase (120-180 s); (c) axial T2-weighted image; (d) axial diffusion-weighted image

$\left(b=1000 \mathrm{~s} / \mathrm{mm}^{2}\right)$; (e) ADC map. T2-weighted images show disruption of the hypointense signal of the sigmoid colon wall and mucosal invasion. There is tumoral enhancement within the lumen. The tumor shows also marked restricted diffusion, which can also be seen in the sigmoid lumen 

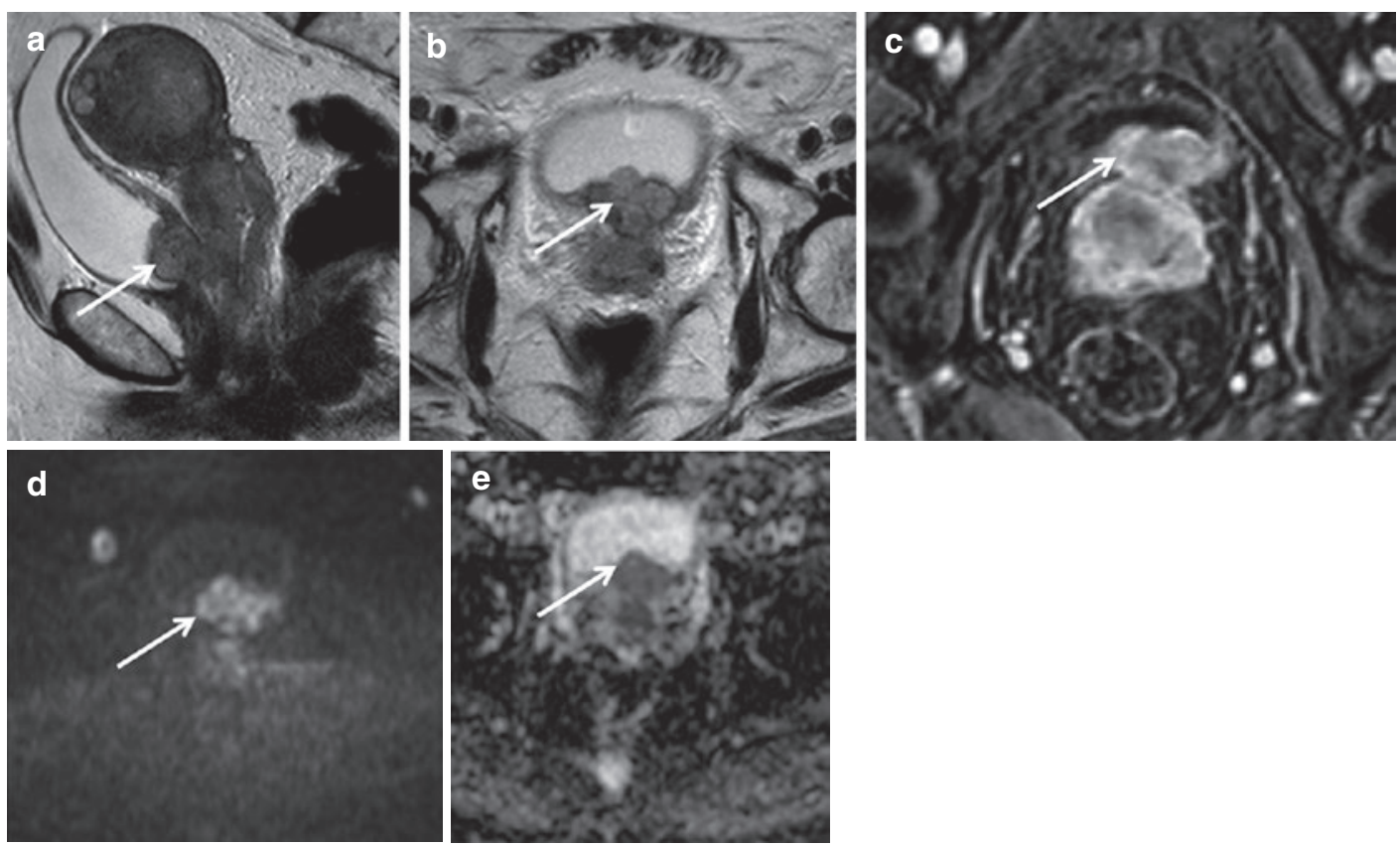

Fig. 26 Stage IVA endometrioid carcinoma that invades the bladder in a 76-year-old woman. (a) Sagittal T2-weighted image; (b) axial T2-weighted image; (c) axial oblique 3D fat-suppressed T1-weighted sequence after the administration of gadolinium in the equilibrium phase $(120 \mathrm{~s})$; (d) axial diffusion-weighted image

\section{$4 \quad$ Therapeutic Approaches}

\subsection{Surgery}

All medical operable patients with endometrial cancer should be considered for surgery (Koh et al. 2014).

The standard surgical approach of endometrial cancer consists of total hysterectomy with bilateral salpingo-oophorectomy, assessment of the abdominal cavity with biopsies of suspicious peritoneal lesions, cytology of peritoneal washing, and at least excision of enlarged lymph nodes (Koh et al. 2014).

Although, systematic pelvic and para-aortic lymphadenopathy is considered part of FIGO surgico-pathological staging, there is still no consensus if it should be performed in cases of treatment of stage I endometrial cancer (Koh et al. 2014; Colombo et al. 2013; Soliman et al. 2010).
( $\left.b=1000 \mathrm{~s} / \mathrm{mm}^{2}\right)$; (e) ADC map. T2-weighted images show disruption of the hypointense signal of the bladder wall and mucosal invasion. There is tumoral enhancement within the lumen. The tumor shows also marked restricted diffusion, which can also be seen in the bladder lumen

A randomized study has shown that in patients where systematic lymphadenectomy was performed, a higher rate of postoperative complications was detected with no improvement of disease-free and overall survival rates (Benedetti Panici et al. 2008). The ASTEC surgical trial also concluded that pelvic lymphadenectomy in patients with early disease did not improve overall or recurrence-free survival (ASTEC study group et al. 2009). These two studies had important limitations and caution must be taken to avoid overinterpretation.

On the other hand, a large retrospective study, the SEPAL study, showed survival benefits in patients with intermediate (FIGO IA and IB, grade 3 endometrioid carcinoma; FIGO IB, grade 1 and 2 endometrioid carcinoma with lymphovascular space invasion; FIGO IC and II endometrioid carcinoma; any type of type 2 endometrial cancer) and high risk (FIGO III and IV endometrioid carcinoma) endometrial carcinoma when 

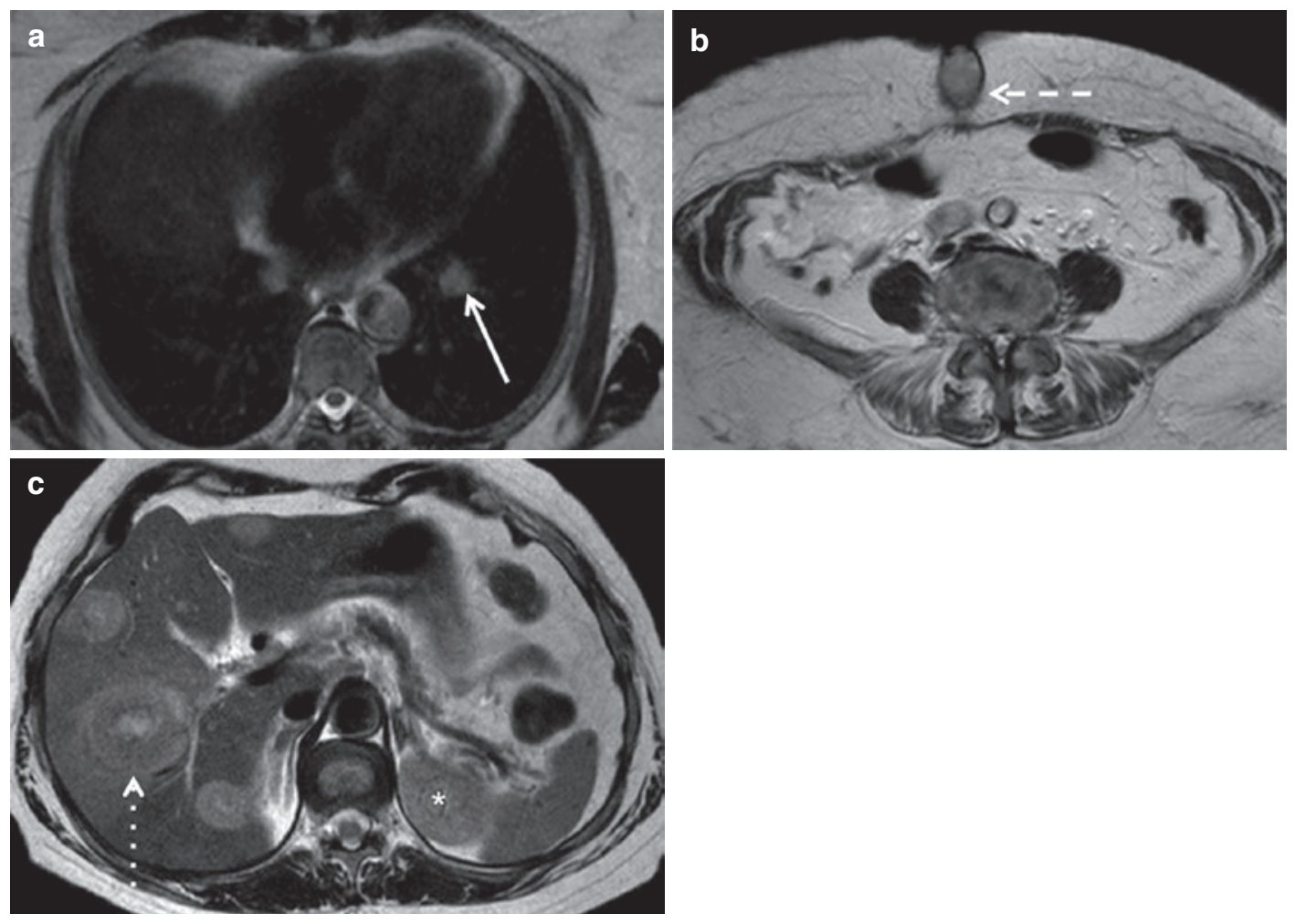

Fig. 27 Stage IVB disease (a) axial T2-weighted image showing a pulmonary metastasis (arrow); (b) axial T2-weighted image showing an umbilical metastasis

submitted to combined pelvic and para-aortic lymphadenectomy (Todo et al. 2010).

Women with stage II should be considered for radical hysterectomy with bilateral salpingooophorectomy and pelvic and para-aortic lymphadenectomy (Colombo et al. 2013).

In patients with a good performance status with advanced stage disease (FIGO stage III and IV), maximum debulking surgery of resettable tumors should be performed. Anterior and posterior pelvic exenteration can be considered for stage IVA disease as well as palliative surgery in patients with metastatic disease (Colombo et al. 2013).

Women with serous, clear-cell carcinomas and carcinosarcomas should be submitted to the same surgical-pathological staging of ovarian cancer (Koh et al. 2014). Serous and clear-cell carcinomas can occur in atrophic endometrium and may present with distant disease even without myometrial invasion; therefore, omentectomy,
(Sister Mary Joseph node) (dashed arrow); (c) axial T2-weighted image showing hepatic metastasis (dotted arrows) and a left adrenal metastasis (asterisk)

diaphragmatic cytology or biopsy, and random peritoneal biopsies should be performed (Koh et al. 2014; Colombo et al. 2013). Pelvic and para-aortic lymphadenectomy is mandatory (Colombo et al. 2013).

\subsection{Adjuvant Treatment}

Radiation therapy is usually used in the adjuvant setting, in the form of external beam radiation therapy (EBRT) and vaginal brachytherapy (VBT).

The purpose of adjuvant radiation therapy is to eliminate microscopic disease in the lymph nodes and in the central pelvic region, including the vaginal cuff; thus reducing the risk of recurrence.

According to the 2014 guidelines of the American Society for Radiation Oncology (ASTRO), patients with endometrioid carcinoma 
that do not require adjuvant radiotherapy (RT) are women with no residual disease in the hysterectomy specimen and patients with grade 1 or 2 endometrial cancer with no myometrial invasion or with $<50 \%$ of myometrial invasion, especially when no high risk features are present (Klopp et al. 2014). Patients with grade 3 endometrioid carcinoma without myometrial invasion and patients with grade 1 or 2 cancer with $<50 \%$ of myometrial invasion and higher risk factors, such as being age $>60$ years and/or lymphovascular space invasion, can be adequately treated with or without VBT (Klopp et al. 2014).

These guidelines also identify the patients with endometrioid cancer for whom vaginal cuff brachytherapy is as effective as pelvic radiation therapy and consequently should be preferred. These patients are women with grade 1 or 2 cancers with $\geq 50 \%$ of myometrial invasion and women with grade 3 tumors with $<50 \%$ of myometrial invasion (Klopp et al. 2014).

In order to decrease pelvic recurrence, the following women with early phase endometrial cancer should receive adjuvant EBRT: patients with stage I, grade 3 cancer with $\geq 50 \%$ of myometrial invasion or patients with cervical stromal invasion; patients with stage I, grade 1 or 2 cancer, with $\geq 50 \%$ of myometrial invasion and other risk factors such as being of age $>60$ years and/or lymphovascular space invasion (Klopp et al. 2014).

If there is a negative prognostic factor, pelvic RT and/or chemotherapy should be performed for stage I and stage II disease (Colombo et al. 2013).

In patients with stage III and IVA disease, external EBRT and adjuvant chemotherapy are justifiable options. These patients can be considered for concurrent chemoradiation followed by adjuvant chemotherapy (Klopp et al. 2014).

If metastatic disease is present, chemotherapyradiotherapy can be performed for palliative treatment (Colombo et al. 2013).

Patients with serous carcinomas, clear-cell carcinomas, and carcinosarcomas should be considered for platinum-based adjuvant chemotherapy with or without radiation therapy (Koh et al. 2014).

\subsection{Fertility-Sparing Treatment}

Fertility-sparing treatment with progestin hormonal therapy may be considered for women who express the desire to preserve fertility and have atypical endometrial hyperplasia or grade 1 endometrioid carcinoma without myometrial involvement in MR (Jafari Shobeiri et al. 2013; Kesterson and Fanning 2012; Colombo et al. 2016). However, this is not the standard of care and the decision to proceed with conservative management is associated with several hazards. Risks include an inadequately staged and treated advanced endometrial cancer; the presence of a synchronous or meta-synchronous cancer; the presence of an inherited genetic predisposition; and the absence of standard medical management and surveillance (Kesterson and Fanning 2012).

\section{$5 \quad$ Follow-Up and Recurrent Endometrial Carcinoma}

The majority of recurrences will occur within the first 3 years $(80 \%)$ (Tirumani et al. 2013). However, only $15 \%$ of patients will develop recurrent disease (Sala et al. 2013; Magrina et al. 2011).

The recommended follow-up is a clinical and gynecological exam at 3-4 months intervals during the first 2 years and then every 6 months until 5 years (Colombo et al. 2013).

During follow-up, radiological exams should only be performed if clinically indicated (Colombo et al. 2013).

Most of endometrial cancer recurrences occur in the lymph nodes (46\%) and in the vaginal vault $(42 \%)$ (Sohaib et al. 2007). The peritoneum and lungs are also reported sites of recurrence, although less frequent (Sohaib et al. 2007).

MR imaging is very useful in diagnosing vaginal vault recurrences, which characteristically display the same signal characteristics as of those of the primary tumor (Tirumani et al. 2013). They tend to appear as a mass disrupting the low signal intensity of the vaginal vault (Sala et al. 2013). MR in these cases plays a role in defining surgical resectability of pelvic recurrences. 
PET-CT has shown high sensitivity (93\%), specificity (93\%), and accuracy (93\%) in detecting recurrent disease (Kitajima et al. 2008). However, more prospective larger studies must determine its role for routine screening (Salani et al. 2011).

\subsection{Treatment of Recurrence}

Treatment of recurrence varies according to the site, to previous treatments, and to the initial stage of the disease.

Vaginal recurrence is the most common and the standard of treatment is EBRT plus VBP (Colombo et al. 2013).

Central pelvic recurrence is usually treated with surgery and radiation therapy, whereas regional pelvic recurrence is treated with radiation therapy plus chemotherapy if possible (Colombo et al. 2013).

Recurrent serous and clear-cell carcinomas should be treated with the same chemotherapeutic regimens of ovarian cancer (Colombo et al. 2013).

\section{Prognosis}

As stated previously, endometrial carcinoma generally has a good outcome. Prognostic factors include histological subtype; histological grade; tumor stage; depth of invasion; and lymphovascular space invasion (Colombo et al. 2013).

Reported 5-year survival rates for stage IA and IB are of $88 \%$ and of $75 \%$, respectively. The prognosis of the different types of stage III substages differs significantly from one another, with estimated 5-year survival rates of $58 \%$ for stage $\mathrm{III}_{\mathrm{C} 1}$ and of $47 \%$ for stage $\mathrm{III}_{\mathrm{C} 2}$. Stage IV disease 5-year survival is estimated at 15-17 \% (American Cancer Society 2016).

The presence of lymph node metastasis, namely para-aortic, has shown to have a strong effect on prognosis (Todo et al. 2010). Tumor grade, lymphovascular space involvement, and the depth of myometrial invasion have been reported as high risk factors for the tumoral involvement of lymph nodes (Patel et al. 2010).

Invasion of the outer half of the myometrium is also strongly associated with a diminished 5-year survival rate (Patel et al. 2010; Amant et al. 2005). Furthermore, it was demonstrated that patients with lymphovascular space involvement had an overall 5-year survival rate of $64 \%$, and on the other hand women without lymphovascular space involvement had an overall 5-year survival rate of $88 \%$ (Colombo et al. 2013).

\section{Conclusion}

Magnetic resonance imaging is not considered in the International Federation of Gynaecology and Obstetrics staging system. However, it plays a relevant role in the preoperative staging of endometrial carcinomas, helping to define the best therapeutic approaches. Moreover, it is important in the diagnosis of treatment complications, in the surveillance of therapy response, and in the assessment of recurrent disease.

Functional MR techniques and specific MR sequence planes play an important role in preventing errors in endometrial cancer staging.

To help prevent diagnostic errors and in order to guide appropriate therapeutic management, radiologists should be aware of common magnetic resonance imaging endometrial appearances for all stages and the frequent mistakes in the assessment of endometrial cancer.

\section{References}

Amant F, Moerman P, Neven P, Timmerman D, Van Limbergen E, Vergote I (2005) Endometrial cancer. Lancet 366(9484):491-505

Amant F, Moerman P, Neven P, Timmerman D, Van Limbergen E, Vergote I (2007) Treatment modalities in endometrial cancer. Curr Opin Oncol 19(5):479-485

American Cancer Society (2016) http://www.cancer.org/ cancer/endometrialcancer/detailedguide/endometrialuterine-cancer-survival-rates. Accessed 25 May 2016

Andreano A, Rechichi G, Rebora P, Sironi S, Valsecchi MG, Galimberti S (2014) MR diffusion imaging for preoperative staging of myometrial invasion in patients with endometrial cancer: a systematic review and 
meta-analysis. Eur Radiol 24(6):1327-1338. doi:10.1007/s00330-014-3139-4

ASTEC Study Group, Kitchener H, Swart AM, Qian Q, Amos C, Parmar MK (2009) Efficacy of systematic pelvic lymphadenectomy in endometrial cancer (MRC ASTEC trial): a randomised study. Lancet 373(9658):125-136. doi:10.1016/ S0140-6736(08)61766-3

Beddy P, Moyle P, Kataoka M, Yamamoto AK, Joubert I, Lomas D et al (2012) Evaluation of depth of myometrial invasion and overall staging in endometrial cancer: comparison of diffusion-weighted and dynamic contrast-enhanced MR imaging. Radiology 262(2): 530-537. doi:10.1148/radiol.11110984

Benedetti Panici P, Basile S, Maneschi F, Alberto Lissoni A, Signorelli M, Scambia G et al (2008) Systematic pelvic lymphadenectomy vs no lymphadenectomy in early-stage endometrial carcinoma: randomized clinical trial. J Natl Cancer Inst 100(23):1707-1716. doi:10.1093/jnci/djn397

Bennet GL, Andreotti RF, Lee SI, Dejesus Allison SO, Brown DL, Dubinsky $\mathrm{T}$ et al (2011 Jul) ACR Appropriateness Criteria ${ }^{\circledR}$ on Abnormal Vaginal Bleeding. J Am Coll Radiol 8(7):460-468. doi:10.1016/j.jacr.2011.03.011

Bharwani N, Miquel ME, Sahdev A, Narayanan P, Malietzis G, Reznek RH et al (2011) Diffusionweighted imaging in the assessment of tumour grade in endometrial cancer. Br J Radiol 84(1007):9971004. doi:10.1259/bjr/14980811

Bonatti M, Stuefer J, Oberhofer N, Negri G, Tagliaferri T, Schifferle $\mathrm{G}$ et al (2015) MRI for local staging of endometrial carcinoma: is endovenous contrast medium administration still needed? Eur J Radiol 84(2):208-214. doi:10.1016/j.ejrad.2014.11.010

Boronow RC (1990) Advances in diagnosis, staging, and management of cervical and endometrial cancer Stages I and II. Cancer 65(3 Suppl):648-659

Briët JM, Hollema H, Reesink N, Aalders JG, Mourits MJE, Hoor ten KA et al (2005) Lymphvascular space involvement: an independent prognostic factor in endometrial cancer. Gynecol Oncol 96(3):799-804

Calle EE, Kaaks R (2004) Overweight, obesity and cancer: epidemiological evidence and proposed mechanisms. Nat Rev Cancer 4(8):579-591

Cancer Incidence Statistics (2015) http://www.cancerresearchuk.org/health-professional/cancer-statistics/ statistics-by-cancer-type/uterine-cancer. Accessed on 22 May 2015

Colombo N, Preti E, Landoni F, Carinelli S, Colombo A, Marini C et al (2013) Endometrial cancer: ESMO Clinical Practice Guidelines for diagnosis, treatment and follow-up. Ann Oncol 24(Suppl 6):vi33-vi38. doi:10.1093/annonc/mdt353

Colombo N, Creutzberg C, Amant F, Bosse T, González-Martín A, Ledermann J et al (2016) ESMO-ESGO-ESTRO consensus conference on endometrial cancer: diagnosis, treatment and followup. Int J Gynecol Cancer 26(1):2-30. doi:10.1097/ IGC.0000000000000609
Creasman W (2009) Revised FIGO staging for carcinoma of the endometrium. Int J Gynaecol Obstet 105(2):109. doi:10.1016/j.ijgo.2009.02.010

Eichhorn JH, Young RH (2001) Neuroendocrine tumors of the genital tract. Am J Clin Pathol 115(Suppl):S94-112

Epstein E, Blomqvist L (2014) Imaging in endometrial cancer. Best Pract Res Clin Obstet Gynaecol 28(5):721-739. doi:10.1016/j.bpobgyn.2014.04.007

Fanning J, Tsukada Y, Piver MS (1990) Intraoperative frozen section diagnosis of depth of myometrial invasion inendometrial adenocarcinoma. Gynecol Oncol 37(1):47-50

Ferlay J, Soerjomataram I, Ervik M, Dikshit R, Eser S, Mathers C, Rebelo M et al (2013) Cancer Incidence and Mortality Worldwide: IARC CancerBase No. 11 [Internet]. Lyon: International Agency for Research on Cancer. Available from: http://globocan.iarc.fr. Accessed 23 May 2015

Fisher B, Costantino JP, Redmond CK, Fisher ER, Wickerham DL, Cronin WM (1994) Endometrial cancer in tamoxifen-treated breast cancer patients: findings from the National Surgical Adjuvant Breast and Bowel Project (NSABP) B-14. J Natl Cancer Inst 86(7):527-537

Frei KA, Kinker K (2001) Staging endometrial cancer: role of magnetic resonance imaging. J Magn Reson Imaging 13(6):850-855

Frei KA, Kinkel K, Bonél HM, Lu Y, Zaloudek C, Hricak $\mathrm{H}$ (2000) Prediction of deep myometrial invasion in patients with endometrial cancer: clinical utility of contrast-enhanced MR imaging-a meta-analysis and bayesian analysis. Radiology 216(2):444-449

Fujii S, Matsusue E, Kigawa J, Sato S, Kanasaki Y, Nakanishi J et al (2008) Diagnostic accuracy of the apparent diffusion coefficient in differentiating benign from malignant uterine endometrial cavity lesions: initial results. Eur Radiol 18(2):384-389

Fujii S, Kido A, Baba T, Fujimoto K, Daido S, Matsumura $N$ (2015) Subendometrial enhancement and peritumoral enhancement for assessing endometrial cancer on dynamic contrast enhanced MR imaging. Eur J Radiol 84(4):581-589. doi:10.1016/j. ejrad.2015.01.004

Gallego JC, Porta A, Pardo MC, Fernández C (2014) Evaluation of myometrial invasion in endometrial cancer: comparison of diffusion-weighted magnetic resonance and intraoperative frozen sections. Abdom Imaging 39(5):1021-1026. doi:10.1007/ s00261-014-0134-9

Goldstein RB, Bree RL, Benson CB, Benacerraf BR, Carlos R et al (2001) Evaluation of the woman with postmenopausal bleeding: society of radiologists in ultrasound-sponsored consensus conference statement. J Ultrasound Med 20(10):1025-1036

Gull B, Karlsson B, Milsom I, Granber S (2003) Can ultrasound replace dilation and curettage? A longitudinal evaluation of postmenopausal bleeding and transvaginal sonographic measurement of the endometrium as predictors of endometrial cancer. Am J Obstet Gynecol 188(2):401-408 
Gupta JK, Chien PF, Voit D, Clark TJ, Khan KS (2002) Ultrasonographic endometrial thickness for diagnosing endometrial pathology in women with postmenopausal bleeding: a meta-analysis. Acta Obstet Gynecol Scand 81(9):799-816

Haider MA, Patlas M, Jhaveri K, Chapman W, Fyles A, Rosen B (2006) Adenocarcinoma involving the uterine cervix: magnetic resonance imaging findings in tumours of endometrial, compared with cervical, origin. Can Assoc Radiol J 57(1):43-48

Haldorsen IS, Salvesen HB (2012) Staging of endometrial carcinomas with MRI using traditional and novel MRI techniques. Clin Radiol 67(1):2-12. doi:10.1016/j. crad.2011.02.018

Haldorsen IS, Grüner R, Husby JA, Magnussen IJ, Werner HMJ, Salvesen $\varnothing \mathrm{O}$ et al (2013) Dynamic contrastenhanced MRI in endometrial carcinoma identifies patients at increased risk of recurrence. Eur Radiol 23(10):2916-2925. doi:10.1007/s00330-013-2901-3

Hecht JL, Mutter GL (2006) Molecular and pathologic aspects of endometrial carcinogenesis. J Clin Oncol 24(29):4783-4791

Hori M, Kim T, Onishi H, Imaoka I, Kagawa Y, Murakami $\mathrm{T}$ et al (2013) Endometrial cancer: preoperative staging using three-dimensional T2-weighted turbo spinecho and diffusion-weighted MR imaging at 3.0 T: a prospective comparative study. Eur Radiol 23(8):2296-2305. doi:10.1007/s00330-013-2815-0

Hulka CA, Hall DA, McCarthy K, Simeone JF (1994) Endometrial polyps, hyperplasia, and carcinoma in postmenopausal women: differentiation with endovaginal sonography. Radiology 191(3):755-758

Ippolito D, Minutolo O, Cadonici A, Talei Franzesi C, Bonaffini P, Perego P et al (2014a) Endometrial cancer: diagnostic value of quantitative measurements of microvascular changes with DCE-MR imaging. MAGMA 27(6):531-538. doi:10.1007/ s10334-014-0435-6

Ippolito D, Cadonici A, Bonaffini PA, Minutolo O, Casiraghi A, Perego P et al (2014b) Semiquantitative perfusion combined with diffusion-weighted MR imaging in pre-operative evaluation of endometrial carcinoma: Results in a group of 57 patients. Magn Reson Imaging 32(5):464-472. doi:10.1016/j. mri.2014.01.009

Jafari Shobeiri M, Mostafa Gharabaghi P, Esmaeili H, Ouladsahebmadarek E, Mehrzad-Sadagiani M (2013) Fertility sparing treatment in young patients with early endometrial adenocarcinoma: case series. Pak J Med Sci 29(2):651-655

Jalloul RJ, Elshaikh MA, Ali-Fehmi R, Haley MM, Yoon J, Mahan M et al (2012) Mucinous adenocarcinoma of the endometrium. Int J Gynecol Cancer 22(5):812818. doi:10.1097/IGC.0b013e31824a

Järvinen HJ, Renkonen-Sinisalo L, Aktán-Collán K, Peltomäki P, Aaltonen LA, Mecklin J-P (2009) Ten years after mutation testing for Lynch syndrome: cancer incidence and outcome in mutation-positive and mutation-negative family members. J Clin Oncol 27(28):4793-4797. doi:10.1200/JCO.2009.23.7784
Kaneda S, Fujii S, Fukunaga T, Kakite S, Kaminou T, Kigawa J et al (2011) Myometrial invasion by endometrial carcinoma: evaluation with 3.0 T MR imaging. Abdom Imaging 36(5):612-618. doi:10.1007/ s00261-011-9719-8

Karlsson B, Granberg S, Wikland M, Ylostalo P, Torvid K, Marsal K et al (1995) Transvaginal ultrasonography of the endometrium in women withpostmenopausal bleeding - a Nordic multicenter study. Am J Obstet Gynecol 172(5):1488-1494

Kesterson JP, Fanning J (2012) Fertility-sparing treatment of endometrial cancer: options, outcomes and pitfalls. J Gynecol Oncol 23(2):120-124. doi:10.3802/ jgo.2012.23.2.120

Kinkel K (2006) Pitfalls in staging uterine neoplasm with imaging: a review. Abdom Imaging 31(2):164-173

Kitajima K, Murakami K, Yamasaki E, Domeki Y, Kaji Y, Morita S et al (2008) Performance of integrated FDG-PET/contrast-enhanced CT in the diagnosis of recurrent uterine cancer: comparison with PET and enhanced CT. Eur J Nucl Med Mol Imaging 36(3):362-372. doi:10.1007/s00259-008-0956-1

Klopp A, Smith BD, Alektiar K, Cabrera A, Damato AL, Erikson B et al (2014) The role of postoperative radiation therapy for endometrial cancer: Executive Summary of an American Society for Radiation Oncology evidence-based guideline. Pract Radiat Oncol 4(3):137-144. doi:10.1016/j. prro.2014.01.003

Koh WJ, Greer BE, Abu-Rustum NR, Apte SM, Campos SM, Chan J et al (2014) Uterine Neoplasms, version 1.2014. J Natl Compr Canc Netw 12(2):248-280

Koyama T, Tamai K, Togashi K (2007) Staging of carcinoma of the uterine cervix and endometrium. Eur Radiol 17(8):2009-2019

Kurman RJ, Carcangiu ML, Herrington CS, Young RH (2014) Tumours of the Uterine Corpus. In: WHO classification of tumours of female reproductive organs, vol 6, 4th edn. International Agency for Research on Cancer, Lyon, pp. 121-155

Larson DM, Connor GP, Broste SK, Krawisz BR, Johnson KK (1996) Prognostic significance of gross myometrial invasion with endometrial cancer. Obstet Gynecol 88(3):394-398

Lopes Dias J, Cunha TM, Gomes FV, Callé C, Félix A (2015) Neuroendocrine tumours of the female genital tract: a case-based imaging review with pathological correlation. Insights Imaging 6(1):43-52. doi:10.1007/ s13244-014-0378-5

Magrina JF, Zanagnolo V, Giles D, Noble BN, Kho RM, Magtibay PM (2011) Robotic surgery for endometrial cancer: comparison of perioperative outcomes and recurrence with laparoscopy, vaginal/laparoscopy and laparotomy. Eur J Gynaecol Oncol 32(5): 476-480

Manfredi R, Mirk P, Maresca G, Margariti PA, Testa A, Zannoni GF et al (2004) Local-regional staging of endometrial carcinoma: role of MR imaging in surgical planning. Radiology 231(2):372-378 
Manfredi R, Gui B, Maresca G, Fanfani F, Bonomo L (2005) Endometrial cancer: magnetic resonance imaging. Abdom Imaging 30(5):626-636

May K, Bryant A, Dickinson HO, Kehoe S, Morrison J (2010) Lymphadenectomy for the management of endometrial cancer. Cochrane Database Syst Rev 20(1):CD007585. doi:10.1002/14651858.CD007585. pub2

McPherson CP, Sellers TA, Potter JD, Bostick RM, Folsom AR (1996) Reproductive factors and risk of endometrial cancer. The Iowa Women's Health Study. Am J Epidemiol 143(12):1195-1202

Meyer LA, Broaddus RR, Lu KH (2009) Endometrial cancer and Lynch syndrome: clinical and pathologic considerations. Cancer Control 16(1):14-22

Nakai G, Matsuki M, Inada Y, Tatsugami F, Tanikake M, Narabayashi I et al (2008) Detection and evaluation of pelvic lymph nodes in patients with gynecologic malignancies using body diffusion-weighted magnetic resonance imaging. J Comput Assist Tomogr 32(5):764-768. doi:10.1097/RCT.0b013e318153fd43

Nakao Y, Yokoyama M, Hara K, Koyamatsu Y, Yasunaga M, Araki Y et al (2006) MR imaging in endometrial carcinoma as a diagnostic tool for the absence of myometrial invasion. Gynecol Oncol 102(2):343-347

Nougaret S, Tirumani SH, Addley H, Pandey H, Sala E, Reinhold C (2013) Pearls and pitfalls in MRI of gynecologic malignancy with diffusion-weighted technique. AJR Am J Roentgenol 200(2):261-276. doi:10.2214/AJR.12.9713

Nougaret S, Reinhold C, Alsharif SS, Addley H, Arceneau J, Molinari N et al (2015) Endometrial cancer: combined MR volumetry and diffusion-weighted imaging for assessment of myometrial and lymphovascular invasion and tumor grade. Radiology 276(3):797-808. doi:10.1148/radiol.15141212

Numazaki R, Miyagi E, Konnai K, Ikeda M, Yamamoto A, Onose R et al (2009) Analysis of stage IVB endometrial carcinoma patients with distant metastasis: a review of prognoses in 55 patients. Int J Clin Oncol 14(4):344-350. doi:10.1007/s10147-009-0878-3

Patel S, Liyanage SH, Sahdev A, Rockall AG, Reznek RH (2010) Imaging of endometrial and cervical cancer. Insights Imaging 1(5-6):309-328

Pecorelli S (2009) Revised FIGO staging for carcinoma of the vulva, cervix, and endometrium. Int J Gynaecol Obstet 105(2):103-104

Rechichi G, Galimberti S, Signorelli M, Perego P, Valsecchi MG, Sironi S (2010) Myometrial invasion in endometrial cancer: diagnostic performance of diffusion-weighted MR imaging at 1.5-T. Eur Radiol 20(3):754-762. doi:10.1007/s00330-009-1597-x

Rechichi G, Galimberti S, Signorelli M, Franzesi CT, Perego P, Valsecchi MG et al (2011) Endometrial cancer: correlation of apparent diffusion coefficient with tumor grade, depth of myometrial invasion, and presence of lymph node metastases. AJR Am J Roentgenol 197(1):256-262. doi:10.2214/ AJR.10.5584
Renehan AG, Tyson M, Egger M, Heller RF, Zwahlen M (2008) Body-mass index and incidence of cancer: a systematic review and meta-analysis of prospective observational studies. Lancet 371(9612):569-578. doi:10.1016/S0140-6736(08)60269-X

Resnick KE, Hampel H, Fishel R, Cohn DE (2009) Current and emerging trends in Lynch syndrome identification in women with endometrial cancer. Gynecol Oncol 114(1):128-134. doi:10.1016/j. ygyno.2009.03.003 Epub 2009 Apr 17

Roy C, Bierry G, Matau A, Bazille G, Pasquali R (2010) Value of diffusion-weighted imaging to detect small malignant pelvic lymph nodes at $3 \mathrm{~T}$. Eur Radiol 20(8):1803-1811. doi:10.1007/s00330-010-1736-4

Saez F, Urresola A, Larena JA, Martín JI, Pijuán JI, Schneider J et al (2000) Endometrial carcinoma: assessment of myometrial invasion with plain and gadolinium-enhanced MR imaging. J Magn Reson Imaging 12(3):460-466

Sala E, Rockall A, Rangarajan D, Kubik-Huch RA (2010) The role of dynamic contrast-enhanced and diffusion weighted magnetic resonance imaging in the female pelvis. Eur J Radiol 76(3):367-385. doi:10.1016/j. ejrad.2010.01.026

Sala E, Rockall AG, Freeman SJ, Mitchell DG, Reinhold C (2013) The added role of MR imaging in treatment stratification of patients with gynecologic malignancies: what the radiologist needs to know. Radiology 266(3):717-740. doi:10.1148/radiol.12120315

Salani R, Backes FJ, Fung MF, Holschneider CH, Parker LP, Bristow RE et al (2011 Jun) Posttreatment surveillance and diagnosis of recurrence in women with gynecologic malignancies: society of Gynecologic Oncologists recommendations. Am J Obstet Gynecol 204(6):466-478. doi:10.1016/j.ajog.2011.03.008

Schmeler KM, Lynch HT, Chen LM, Munsell MF, Soliman PT, Clark MB et al (2006) Prophylactic surgery to reduce the risk of gynecologic cancers in the lynch syndrome. N Engl J Med 354(3):261-269

Scoutt LM, McCarthy SM, Flynn SD, Lange RC, Long F, Smith RC et al (1995) Clinical stage I endometrial carcinoma: pitfalls in preoperative assessment with MR imaging Work in progress. Radiology 194(2): $567-572$

Seki H, Takano T, Sakai K (2000) Value of dynamic MR imaging in assessing endometrial carcinoma involvement of the cervix. AJR Am J Roentgenol 175(1):171-176

Setiawan VW, Yang HP, Pike MC, McCann SE, Yu H, Xiang Y-B et al (2013) Type I and II endometrial cancers: have they different risk factors? J Clin Oncol 31(20):2607-2618. doi:10.1200/JCO.2012.48.2596

Shapiro S, Kelly JP, Rosenberg L, Kaufman DW, Helmrich SP, Rosenshein NB et al (1985) Risk of localized and widespread endometrial cancer in relation to recent and discontinued use of conjugated estrogens. N Engl J Med 313(16):969-972

Shen S-H, Chiou Y-Y, Wang J-H, Yen M-S, Lee R-C, Lai C-R et al (2008) Diffusion-weighted single-shot echoplanar imaging with parallel technique in assessment 
of endometrial cancer. Am J Roentgenol 190(2):481488. doi:10.2214/AJR.07.2155

Smith-Bindman R, Kerlikowske K, Feldstein VA, Subak L, Scheidler J, Segal M et al (1998) Endovaginal ultrasound to exclude endometrial cancer and other endometrial abnormalities. JAMA 280(17):1510-1517

Sohaib SA, Houghton SL, Meroni R, Rockall AG, Blake P, Reznek RH (2007) Recurrent endometrial cancer: patterns of recurrent disease and assessment of prognosis. Clin Radiol 62(1):28-34

Soliman PT, Wu D, Tortolero-Luna G, Schmeler KM, Slomovitz BM, Bray MS et al (2006) Association between adiponectin, insulin resistance, and endometrial cancer. Cancer 106(11):2376-2381

Soliman PT, Frumovitz M, Spannuth W, Greer MJ, Sharma S, Schmeler KM et al (2010 Nov) Lymphadenectomy during endometrial cancer staging: Practice patterns among gynecologic oncologists. Gynecol Oncol 119(2):291-294. doi:10.1016/j.ygyno.2010.07.011

Takeuchi M, Matsuzaki K, Nishitani H (2009) Diffusion-weighted magnetic resonance imaging of endometrial cancer: differentiation from benign endometrial lesions and preoperative assessment of myometrial invasion. Acta Radiol 50(8):947-953. doi:10.1080/02841850903099981

Tamai K, Koyama T, Saga T, Umeoka S, Mikami Y, Fujii $S$ et al (2007) Diffusion-weighted MR imaging of uterine endometrial cancer. J Magn Reson Imaging 26(3):682-687

Tirumani SH, Shanbhogue AK, Prasad SR (2013) Current concepts in the diagnosis and management of endometrial and cervical carcinomas. Radiol Clin North Am 51(6):1087-1110. doi:10.1016/j.rcl.2013.07.003

Todo Y, Kato H, Kaneuchi M, Watari H, Takeda M, Sakuragi N (2010) Survival effect of para-aortic lymphadenectomy in endometrial cancer (SEPAL study): a retrospective cohort analysis. Lancet 375(9721):11651172. doi:10.1016/S0140-6736(09)62002-X

Vargas HA, Akin O, Zheng J, Moskowitz C, Soslow R, AbuRustum N et al (2011) The value of MR imaging when the site of uterine cancer origin is uncertain. Radiology 258(3):785-792. doi:10.1148/radiol.10101147

Venkat P, Chen LM, Young-Lin N, Kiet TK, Young G, Amatori D et al (2012) An economic analysis of robotic versus laparoscopic surgery for endometrial cancer: costs, charges and reimbursements to hospitals and professionals. Gynecol Oncol 125(1):237-240. doi:10.1016/j.ygyno.2011.11.036

Wakefield JC, Downey K, Kyriazi S, deSouza NM (2013) New MR techniques in gynecologic cancer. AJR Am J Roentgenol 200(2):249-260. doi:10.2214/ AJR.12.8932

Yamashita Y, Mizutani H, Torashima M, Takahashi M, Miyazaki K, Okamura H et al (1993a) Assessment of myometrial invasion by endometrial carcinoma: transvaginal sonography vs contrast-enhanced MR imaging. AJR Am J Roentgenol 161(3):595-599

Yamashita Y, Harada M, Sawada T, Takahashi M, Miyazaki K, Okamura H (1993b) Normal uterus and FIGO stage I endometrial carcinoma: dynamic gadolinium-enhanced MR imaging. Radiology 186(2):495-501 